\title{
Kinetic formulation for heterogeneous scalar conservation laws
}

\section{Formulation cinétique des lois de conservation scalaires hétérogènes}

\author{
Anne-Laure Dalibard \\ CEREMADE-UMR 7534, Université Paris-Dauphine, Place du maréchal de Lattre de Tassigny, 75775 Paris cedex 16, France
}

Received 22 April 2005; accepted 20 May 2005

Available online 2 December 2005

\begin{abstract}
This article is concerned with the study of entropy solutions of the scalar conservation law $\partial_{t} u+\operatorname{div}_{x} A(x, u)=0$. The main novelty of our work lies in the fact that the flux $A$ is allowed to depend on the space variable $x$. We introduce a kinetic formulation for this equation, proven to be equivalent to the usual entropy inequalities. We check the existence and uniqueness of weak $L^{1}$ solutions of the scalar law.

C 2006 L'Association Publications de l'Institut Henri Poincaré. Published by Elsevier B.V. All rights reserved
\end{abstract}

\section{Résumé}

On étudie ici les solutions entropiques de lois de conservation scalaires hétérogènes $\partial_{t} u+\operatorname{div}_{x} A(x, u)=0$. L'apport principal de notre étude vient de la forme plus générale du flux $A$, qui dépend explicitement de la variable d'espace $x$. On commence par introduire une formulation cinétique de la loi de conservation, dont on montre qu'elle est équivalente aux inégalités entropiques habituelles. On vérifie ensuite l'existence et l'unicité de solutions faibles $L^{1}$ de la loi de conservation.

(C) 2006 L'Association Publications de l'Institut Henri Poincaré. Published by Elsevier B.V. All rights reserved

MSC: $35 \mathrm{~L} 65$

Keywords: Scalar conservation law; Entropy solutions; Kinetic formulation

\section{Introduction}

We are interested in the properties of scalar functions $u(t, x)$ solutions in the sense of distributions of the multidimensional scalar conservation law:

$$
\left\{\begin{array}{l}
\frac{\partial u}{\partial t}(t, x)+\sum_{i=1}^{n} \frac{\partial}{\partial x_{i}} A_{i}(x, u(t, x))=0, \quad t \geqslant 0, x \in \mathbb{R}^{n}, \\
u(t=0, x)=u^{0}(x) \in L^{1}\left(\mathbb{R}^{n}\right) \cap L^{\infty}\left(\mathbb{R}^{n}\right),
\end{array}\right.
$$

with $n \in \mathbb{N}$ arbitrary.

E-mail address: dalibard@ ceremade.dauphine.fr (A.-L. Dalibard). 
Many fundamental contributions to the theory of conservation laws already exist (see for instance [1,10], and the references therein), mostly in homogeneous cases, i.e. when the flux $A$ does not depend on the space variable $x$.

Following an idea of P.-L. Lions, B. Perthame and E. Tadmor ([5,6]; see also the presentation of B. Perthame in [7]), we introduce a kinetic formulation for the problem (1) and we prove the existence and uniqueness of entropy solutions. The main contribution of this work is the more general form of the flux $A$ : we wish to emphasize the fact that the spatial dependence of the flux $A$ will require to modify substantially the kinetic formulation developed in the homogeneous case. From a physical point of view, the $x$-dependence of the flux $A$ accounts for spatial heterogeneities.

Let us precise the regularity assumptions we will need on the flux $A_{i}$ : the minimal hypotheses are

$$
\begin{aligned}
& a_{i}(x, v):=\frac{\partial A_{i}}{\partial v}(x, v) \in L_{\mathrm{loc}}^{\infty}\left(\mathbb{R} ; L^{\infty}\left(\mathbb{R}^{n}\right)\right), \\
& a_{n+1}(x, v):=-\sum_{i=1}^{n} \frac{\partial A_{i}}{\partial x_{i}}(x, v) \in L^{\infty}\left(\mathbb{R}^{n+1}\right), \\
& a_{n+1}(x, 0)=0 \quad \forall x \in \mathbb{R}^{n} .
\end{aligned}
$$

The proofs of existence and uniqueness require more regularity, namely

$$
\begin{aligned}
& a_{i} \in \mathcal{C}^{1}\left(\mathbb{R}^{n+1}\right), \quad \partial_{j} a_{i} \in L_{\mathrm{loc}}^{\infty}\left(\mathbb{R} ; L^{\infty}\left(\mathbb{R}^{n}\right)\right), \quad 1 \leqslant i, j \leqslant n+1, \\
& \exists C>0, \quad \forall(x, v) \in \mathbb{R}^{n+1}, \quad\left|a_{i}(x, v)\right| \leqslant C(1+|x|+|v|), \quad 1 \leqslant i \leqslant n+1
\end{aligned}
$$

for the proof of existence and

$$
\partial_{j} a_{i} \in L_{\mathrm{loc}}^{1}\left(\mathbb{R}^{n+1}\right), \quad 1 \leqslant i, j \leqslant n+1
$$

(with the convention $\partial_{j}=\partial_{x_{j}}$ for $1 \leqslant j \leqslant n$, and $\partial_{n+1}=\partial_{v}$ ) for the proof of uniqueness.

These assumptions could probably be weakened, but are rather convenient to work with in a first step. Notice that condition (4) is not very restrictive: indeed, if (4) is false and $u$ is a solution of (1), then setting $B_{i}(x, v)=$ $A_{i}(x, v)-A_{i}(x, 0), u$ is a solution of

$$
\frac{\partial u}{\partial t}+\sum_{i=1}^{n} \frac{\partial}{\partial x_{i}} B_{i}(x, u(t, x))=a_{n+1}(x, 0)
$$

$B$ satisfies assumption (4); thus, the only change in the analysis is the addition of a source term. Remark also that (4) is satisfied if and only if the null function is a solution of (1).

We denote by $a=a(x, v)$ the vector valued function $a:=\left(a_{1}, \ldots, a_{n}, a_{n+1}\right)$. Notice that $\operatorname{div}_{x, v} a=0$. This divergence-free property will be fundamental in the proofs and, in some sense, replaces the fact that $a$ is independent of $x$ and $a_{n+1}=0$ in the homogeneous case.

The organization of the article is as follows: in the second section, we recall the definition of an entropy solution and show the equivalence between the family of entropy inequalities and the kinetic formulation. This equivalence allows us to define a weaker notion of solutions, called kinetic solutions. In the third section, the existence of kinetic solutions of (1) is proved. In the fourth and last section, we show the uniqueness of kinetic solutions.

\section{Entropy solutions and kinetic formulation}

\subsection{Different notions of solutions - main results}

Let us recall the notion of entropy solution introduced by Kruzkhov (see [3,4]):

Definition 1. A function $u \in \mathcal{C}\left(\mathbb{R}_{+}, L^{1}\left(\mathbb{R}^{n}\right)\right) \cap L_{\text {loc }}^{\infty}\left(\mathbb{R}_{+}, L^{\infty}\left(\mathbb{R}^{n}\right)\right)$ is an entropy solution of the system (1) if it is a solution of (1) in the distributional sense, and if it satisfies the following inequality for all convex functions $S \in \mathcal{C}^{2}(\mathbb{R})$ :

$$
\frac{\partial S(u)}{\partial t}+\sum_{i=1}^{n} \frac{\partial}{\partial x_{i}} \eta_{i}(x, u)+\sum_{i=1}^{n} \frac{\partial A_{i}}{\partial x_{i}}(x, u) S^{\prime}(u)-\sum_{i=1}^{n} \frac{\partial \eta_{i}}{\partial x_{i}}(x, u) \leqslant 0,
$$


where

$$
\eta_{i}(x, v):=\int_{0}^{v} a_{i}\left(x, v^{\prime}\right) S^{\prime}\left(v^{\prime}\right) \mathrm{d} v^{\prime} .
$$

The inequality (8) will be called entropy inequality, and the whole family of entropy inequalities will be referred to as entropy formulation.

The following result, due to Kruzkhov (see $[3,4]$ ) states that the Cauchy problem is well-posed in this class of solutions:

Proposition 2. Let $u^{0} \in L^{1}\left(\mathbb{R}^{n}\right) \cap L^{\infty}\left(\mathbb{R}^{n}\right)$.

Under the assumptions $A \in \mathcal{C}\left(\mathbb{R}^{n+1}\right), a_{i} \in \mathcal{C}\left(\mathbb{R}^{n+1}\right) \cap L_{\mathrm{loc}}^{\infty}\left(\mathbb{R} ; L^{\infty}\left(\mathbb{R}^{n}\right)\right)$ for $1 \leqslant i \leqslant n, a_{n+1} \in L_{\mathrm{loc}}^{\infty}\left(\mathbb{R} ; L^{\infty}\left(\mathbb{R}^{n}\right)\right)$, $\frac{\partial a_{i}}{\partial x_{j}} \in \mathcal{C}\left(\mathbb{R}^{n+1}\right)(1 \leqslant i \leqslant n+1,1 \leqslant j \leqslant n)$, there exists a unique entropy solution $u \in \mathcal{C}\left([0,+\infty), L^{1}\left(\mathbb{R}^{n}\right)\right) \cap$ $L_{\text {loc }}^{\infty}\left(\mathbb{R}_{+}, L^{\infty}\left(\mathbb{R}^{n}\right)\right)$ of the scalar conservation law (1) with initial data $u^{0}$.

Moreover, if $u, v$ are entropy solutions with initial data $u^{0}$ and $v^{0}$, then $u$ and $v$ satisfy the $L^{1}$ contraction property:

$$
\|u(t)-v(t)\|_{L^{1}} \leqslant\left\|u^{0}-v^{0}\right\|_{L^{1}} \quad \forall t \in \mathbb{R}_{+} .
$$

Our aim in this paper is in to give a better understanding of the entropy solutions of (1) by introducing a kinetic formulation. This will allow us to generalize Kruzkhov's result and to develop a pure $L^{1}$ setting for solutions of (1).

Define $\chi: \mathbb{R}^{2} \rightarrow\{1,-1,0\}$ as follows:

$$
\chi(v, u):= \begin{cases}1 & \text { if } 0<v<u \\ -1 & \text { if } u<v<0 \\ 0 & \text { otherwise }\end{cases}
$$

Our first result is the following:

Theorem 3. Let $u \in \mathcal{C}\left([0,+\infty), L^{1}\left(\mathbb{R}^{n}\right)\right) \cap L_{\text {loc }}^{\infty}\left((0,+\infty), L^{\infty}\left(\mathbb{R}^{n}\right)\right)$. Under the assumptions (2)-(4), $u$ is an entropy solution of (1) if and only if there exists a nonnegative measure $m(t, x, v)$ such that $m\left((0, T) \times \mathbb{R}^{n+1}\right)<+\infty$ for all $T>0$, and such that $\chi(v, u(t, x))$ satisfies the following kinetic equation in the sense of distributions on $\mathbb{R}_{+} \times \mathbb{R}^{n+1}$ :

$$
\left\{\begin{array}{l}
\partial_{t} \chi(v, u(t, x))+\operatorname{div}_{x, v}[a(x, v) \chi(v, u(t, x))]=\partial_{v} m(t, x, v), \\
\chi(v, u(t=0, x))=\chi\left(v, u^{0}(x)\right) .
\end{array}\right.
$$

We shall say that Eq. (10) is the kinetic formulation of the scalar conservation law (1).

Remark 4. The kinetic formulation of Eq. (1) will prove to be much more handy than the equation itself due to the linearity of Eq. (10). However, it should be noticed that the nonlinearity is somewhat hidden in Eq. (10). Note indeed that in addition to the introduction of an extra "fluctuation" variable $v$, the function $\chi$ is nonlinear. The measure $m$ is called the entropy defect measure and accounts for the lack of regularity of $u: m$ vanishes on sets of $\mathbb{R}_{+} \times \mathbb{R}^{n}$ on which $u$ is smooth (say $W^{1,1}$ for instance).

This result allows us to define a weaker notion of solution:

Definition 5. Let $u \in \mathcal{C}\left([0,+\infty), L^{1}\left(\mathbb{R}^{n}\right)\right)$. $u$ is a kinetic solution of (1) if there exists a nonnegative measure $m$ such that $m\left((0, T) \times \mathbb{R}^{n} \times(-R, R)\right)$ is finite for all $T, R>0$ and such that (10) is satisfied in the sense of distributions.

We will prove that the Cauchy problem is also well-posed in this class of solutions:

Theorem 6. Let $u^{0} \in L^{1}\left(\mathbb{R}^{n}\right)$. Under the assumptions (2)-(6), there exists a kinetic solution $u \in \mathcal{C}\left([0,+\infty), L^{1}\left(\mathbb{R}^{n}\right)\right)$ of the scalar conservation law (1) such that $u(t=0)=u^{0}$. 
Moreover, under assumptions (2)-(4), (7) if $u_{1}, u_{2}$ are two kinetic solutions of (1) such that for all $T>0$

$$
\int_{0}^{T} \int_{\mathbb{R}^{n+1}}\left|a_{i}(x, v)\right|\left|\chi\left(v, u_{j}(t, x)\right)\right| \mathrm{d} x \mathrm{~d} v \mathrm{~d} t<+\infty, \quad 1 \leqslant i \leqslant n, j=1,2,
$$

then the $L^{1}$ contraction property holds:

$$
\left\|u_{1}(t)-u_{2}(t)\right\|_{L^{1}\left(\mathbb{R}^{n}\right)} \leqslant\left\|u_{1}(t=0)-u_{2}(t=0)\right\|_{L^{1}\left(\mathbb{R}^{n}\right)} \quad \forall t>0 .
$$

Remark 7. In fact, uniqueness will be proved in a broader class of solutions, called generalized kinetic solutions (see Section 4).

Remark 8. Pushing further the equivalence between entropy and kinetic solutions, it can be proved that $u \in$ $\mathcal{C}\left([0,+\infty), L^{1}\left(\mathbb{R}^{n}\right)\right)$ is a kinetic solution of (1) if and only if $u$ is a solution of (1) in the distributional sense and if there exists a nonnegative measure $m_{0}(t, x, v)$ such that the following equality holds for all functions $S \in \mathcal{C}^{2}(\mathbb{R})$ such that $S^{\prime}$ has bounded support

$$
\begin{aligned}
\frac{\partial}{\partial t} & S(u(t, x))+\sum_{i=1}^{n} \frac{\partial}{\partial x_{i}} \eta_{i}(x, u(t, x))+\sum_{i=1}^{n} \frac{\partial A_{i}}{\partial x_{i}}(x, u) S^{\prime}(u)-\sum_{i=1}^{n} \frac{\partial \eta_{i}}{\partial x_{i}}(x, u) \\
& =-\int_{\mathbb{R}} S^{\prime \prime}(v) m_{0}(t, x, v) \mathrm{d} v .
\end{aligned}
$$

In that case, $m$ (the kinetic entropy defect measure) and $m_{0}$ are equal, up to a function of $t, x$.

The proof of this result is the same as the one of Theorem 3, and is left to the reader.

In the following subsection, we prove Theorem 3. Sections 3 and 4 are devoted to the proof of Theorem 6: more precisely, Section 3 is concerned with the proof of the existence, and Section 4 with the one of uniqueness.

\subsection{Equivalence between the entropy and kinetic formulations - proof of Theorem 3}

The equivalence of initial conditions is easily checked; concerning the equivalence of the evolution equations, we define a distribution $m$ which is a solution of (10), and we prove that $m$ is nonnegative and locally bounded if and only if $u$ is an entropy solution.

First step: definition of $m$.

Let $u \in \mathcal{C}\left(\mathbb{R}_{+}, L^{1}\left(\mathbb{R}^{n}\right)\right) \cap L_{\text {loc }}^{\infty}\left(\mathbb{R}_{+}, L^{\infty}\left(\mathbb{R}^{n}\right)\right)$. Define the distribution $m(t, x, v)$ by

$$
m(t, x, v):=\frac{\partial}{\partial t} \int_{0}^{v} \chi(w, u(t, x)) \mathrm{d} w+\frac{\partial}{\partial x_{i}} \int_{0}^{v} a_{i}(x, w) \chi(w, u(t, x)) \mathrm{d} w+a_{n+1}(x, v) \chi(v, u(t, x)),
$$

so that (10) is satisfied in the sense of distributions.

We multiply (10) by $S^{\prime}(v)$, with $S^{\prime} \in \mathcal{D}(\mathbb{R})$, and integrate with respect to $v$. We shall use systematically the following easy fact

$$
\int f^{\prime}(v) \chi(v, u) \mathrm{d} v=f(u)-f(0) \quad \forall u \in \mathbb{R} \quad \forall f \in W_{\text {loc }}^{1, \infty}(\mathbb{R}) .
$$

Taking $f=S$ and $f=\eta_{i}$, and using the following identity (recall that (4) is satisfied)

$$
\frac{\partial A_{i}}{\partial x_{i}}(x, v) S^{\prime}(v)=\frac{\partial \eta_{i}}{\partial x_{i}}(x, v)-\int_{\mathbb{R}} \chi(w, v) a_{n+1}(x, w) S^{\prime \prime}(w) \mathrm{d} w \quad \forall(x, v) \in \mathbb{R}^{n+1}
$$

leads us to the following equation, which holds true as long as $S^{\prime} \in \mathcal{D}(\mathbb{R})$ : 


$$
\frac{\partial}{\partial t} S(u(t, x))+\sum_{i=1}^{n} \frac{\partial}{\partial x_{i}} \eta_{i}(x, u(t, x))+\sum_{i=1}^{n} \frac{\partial A_{i}}{\partial x_{i}}(x, u) S^{\prime}(u)-\sum_{i=1}^{n} \frac{\partial \eta_{i}}{\partial x_{i}}(x, u)=-\int_{\mathbb{R}} S^{\prime \prime}(v) m(t, x, v) \mathrm{d} v .
$$

Second step: If $m \geqslant 0$ is a locally bounded measure, then $u$ is an entropy solution of (1).

Assume $m$ is a nonnegative measure on $\mathbb{R}_{+} \times \mathbb{R}^{n+1}$ such that

$$
\int_{0}^{T} \int_{\mathbb{R}^{n+1}} m(t, x, v) \mathrm{d} t \mathrm{~d} x \mathrm{~d} v<\infty \quad \forall T>0 .
$$

In the sequel, we will work on a compact time interval $[0, T]$, and we set

$$
M=\|u\|_{L^{\infty}\left((0, T) \times \mathbb{R}^{n}\right)}<\infty .
$$

First, let us generalize (15) to all $S \in \mathcal{C}^{2}(\mathbb{R})$ subquadratic, i.e. such that $S^{\prime \prime}$ is bounded. If $S \in \mathcal{C}^{\infty}(\mathbb{R})$ is subquadratic, we truncate $S$ thanks to a cut-off function $\phi \in \mathcal{C}_{0}^{\infty}(\mathbb{R})$ s.t. $\phi(v) \equiv 1$ for $|v| \leqslant 1$ and $\phi(v) \equiv 0$ for $|v| \geqslant 2$ : set $\phi_{k}(v)=\phi\left(\frac{v}{k}\right), S_{k}=S \phi_{k}$. Then $S_{k} \in \mathcal{D}(\mathbb{R})$, and we may write (15) for $S_{k}$.

But for $k>M, S_{k}(u(t, x))=S(u(t, x))$ and $\eta_{k}(x, u)=\eta(x, u)$ for almost every $(t, x)$. Thus, for $k>M$, on $\mathcal{D}^{\prime}\left((0, T) \times \mathbb{R}^{n}\right)$, we have

$$
\frac{\partial}{\partial t} S(u(t, x))+\sum_{i=1}^{n} \frac{\partial}{\partial x_{i}} \eta_{i}(x, u(t, x))+\sum_{i=1}^{n} \frac{\partial A_{i}}{\partial x_{i}}(x, u) S^{\prime}(u)-\sum_{i=1}^{n} \frac{\partial \eta_{i}}{\partial x_{i}}(x, u)=-\int_{\mathbb{R}} S_{k}^{\prime \prime}(v) m(t, x, v) \mathrm{d} v .
$$

Then, we pass to the limit as $k \rightarrow \infty$ thanks to the bounds on $S^{\prime \prime}$ and $m$. Eq. (15) is thus true for all functions $S \in \mathcal{C}^{\infty}(\mathbb{R})$ subquadratic, which implies in particular that $u$ is a distributional solution of (1). In a similar fashion, equality (15) is extended to $S \in \mathcal{C}^{2}(\mathbb{R})$ convex and subquadratic by mollifying $S$ and passing to the limit thanks to the bounds on $S, m$, and $u$.

Now, let $S \in \mathcal{C}^{2}(\mathbb{R})$ convex. Since $u$ is bounded on $(0, T) \times \mathbb{R}^{n}$, we can change the values of $S(v)$ for large $v$ in order to obtain a subquadratic function: set for instance $C=\left\|S^{\prime \prime}\right\|_{L^{\infty}(-M, M)}$ and define the $\mathcal{C}^{2}$ convex subquadratic function $\widetilde{S}$ such that $\widetilde{S}^{\prime \prime}=\inf \left(C, S^{\prime \prime}\right)$ and $\widetilde{S}=S$ on $[-M, M]$. We have $S(u)=\widetilde{S}(u)$ a.e. in $(0, T) \times \mathbb{R}^{n}$ (idem with $\eta_{i}$ and $S^{\prime}$ ); moreover, $\widetilde{S}$ satisfies Eq. (15), and thus inequality (8), since $\widetilde{S}$ is convex. Thus, (8) is true for all convex functions $S \in \mathcal{C}^{2}(\mathbb{R})$ and $u$ is an entropy solution of (1).

Third step: If $u$ is an entropy solution of (1), then $m$ is a nonnegative locally bounded measure.

Let us now prove the reciprocal implication. First, remark that if $S \in \mathcal{C}^{\infty}(\mathbb{R})$ is a convex function such that $S^{\prime} \in \mathcal{D}(\mathbb{R})$, then comparing (8) and (15), we have

$$
\int_{\mathbb{R}} S^{\prime \prime}(v) m(t, x, v) \mathrm{d} v \geqslant 0 .
$$

Unfortunately, if we consider any nonnegative test function $\varphi \in \mathcal{D}(\mathbb{R})_{+}$, we cannot find $S$ such that $S^{\prime \prime}=\varphi$ and $S^{\prime} \in \mathcal{D}(\mathbb{R})$. The idea is thus to change the values of $\varphi(v)$ for large $v$ in order to be able to find such a function $S$. This requires to investigate the behavior of $m$ for large $v$.

Indeed, for $|v|>M$, Eq. (13) becomes

$$
\frac{\partial}{\partial t} u(t, x)+\sum_{i=1}^{n} \frac{\partial}{\partial x_{i}} A_{i}(x, u(t, x))=m(t, x, v) .
$$

Comparing the above equation to (1) yields

$$
m(t, x, v)=0 \quad \text { in } \mathcal{D}^{\prime}\left((0, T) \times \mathbb{R}^{n} \times(M,+\infty)\right) .
$$

Now, if $\varphi \in \mathcal{D}(\mathbb{R})_{+}$is an arbitrary nonnegative test function, define $S \in \mathcal{C}^{\infty}$ such that $S^{\prime \prime}=\varphi$; $S^{\prime \prime}$ is a convex function. Multiplying $S$ by a cut-off function which is equal to 1 over the interval $[-M, M]$, and denoting by $\widetilde{S} \in \mathcal{D}(\mathbb{R})$ the function thus obtained, we have:

(1) $S(u(t, x))=\widetilde{S}(u(t, x))$ on $(0, T) \times \mathbb{R}^{n}$ (idem for $\eta_{i}$ and $\left.S^{\prime}\right)$; 
(2) $\int_{\mathbb{R}} \varphi(v) m(t, x, v) \mathrm{d} v=\int_{\mathbb{R}} \widetilde{S}^{\prime \prime}(v) m(t, x, v) \mathrm{d} v$;

(3) $\widetilde{S}$ satisfies (15);

(4) $S$ satisfies (8).

Combining all these properties leads us to

$$
\int_{\mathbb{R}} \varphi(v) m(t, x, v) \mathrm{d} v \geqslant 0 \quad \forall \varphi \in \mathcal{D}(\mathbb{R})_{+} .
$$

Thus, $m$ is a nonnegative distribution (and hence a nonnegative measure) on $\mathbb{R}_{+} \times \mathbb{R}^{n+1}$.

Finally, let us show that for all $T>0$,

$$
\int_{0}^{T} \int_{\mathbb{R}^{n+1}} m(t, x, v) \mathrm{d} t \mathrm{~d} x \mathrm{~d} v \leqslant \frac{1}{2}\left\|u^{0}\right\|_{L^{2}\left(\mathbb{R}^{n}\right)}^{2}+\int_{0}^{T} \int_{\mathbb{R}^{n+1}} a_{n+1}(x, v) \chi(v, u(t, x)) \mathrm{d} t \mathrm{~d} x \mathrm{~d} v .
$$

Indeed, thanks to (17), (15) is true for all convex $\mathcal{C}^{2}$ functions (the proof of this fact is the same as in the first part of the equivalence). Write (15) for $S \in \mathcal{C}^{2}$ a nonnegative convex subquadratic function such that $S(0)=0$; then $S(u(t, \cdot)) \in L^{1}\left(\mathbb{R}^{n}\right)$ for all $t>0$. Integrating $(15)$ on $(0, T) \times \mathbb{R}^{n}$ yields

$$
\int_{0}^{T} \int_{\mathbb{R}^{n+1}} S^{\prime \prime}(v) m(t, x, v) \mathrm{d} v \mathrm{~d} x \mathrm{~d} t \leqslant \int_{\mathbb{R}^{n}} S\left(u^{0}(x)\right) \mathrm{d} x+\int_{0}^{T} \int_{\mathbb{R}^{n+1}} a_{n+1}(x, v) \chi(v, u(t, x)) S^{\prime \prime}(v) \mathrm{d} v \mathrm{~d} x \mathrm{~d} t .
$$

The choice $S(v)=\frac{|v|^{2}}{2}$ gives the desired result.

Remark 9. We wish to lay emphasis on a major difference with homogeneous scalar conservation laws. For a homogeneous conservation law, if $S \in \mathcal{C}^{2}(\mathbb{R})$ is convex and such that $S(0)=0$, then for any entropy solution in $\mathcal{C}\left(\mathbb{R}_{+}, L^{1}\left(\mathbb{R}^{n}\right)\right) \cap L^{\infty}\left(\mathbb{R}_{+} \times \mathbb{R}^{n}\right)$

$$
\frac{\mathrm{d}}{\mathrm{d} t} \int_{\mathbb{R}^{n}} S(u(t, x)) \mathrm{d} x \leqslant 0 .
$$

In particular, all $L^{p}$ norms are nonincreasing (see [7]).

This is no longer true for heterogeneous conservation laws: indeed, integrating formally (8) on $\mathbb{R}^{n}$ yields

$$
\frac{\mathrm{d}}{\mathrm{d} t} \int_{\mathbb{R}^{n}} S(u(t, x)) \mathrm{d} x \leqslant \int_{\mathbb{R}^{n+1}} S^{\prime \prime}(v) a_{n+1}(x, v) \chi(v, u(t, x)) \mathrm{d} x \mathrm{~d} v .
$$

In general, the right-hand side of the above inequality is not nonpositive, and no $L^{p}$ norm is conserved for $p>1$. If we want to retrieve the properties of homogeneous laws, we need to take additional hypotheses on the flux $A$ in order that

$$
\int_{\mathbb{R}^{n}} a_{n+1}(x, v) \chi(v, u(t, x)) \mathrm{d} x \leqslant 0 .
$$

In particular, this is the case if we assume

$$
\operatorname{sgn}(v) a_{n+1}(v)=-\left|a_{n+1}(v)\right| \leqslant 0 .
$$

Notice that this hypothesis is satisfied for homogeneous fluxes (in that case $a_{n+1} \equiv 0$ ).

It can be shown under the assumptions of Theorem 2 and (21) that all entropy solutions of (1) satisfy (20). And if $m$ is the kinetic entropy defect measure, then $m\left((0,+\infty) \times \mathbb{R}^{n+1}\right)<\infty$. 


\section{Existence of kinetic solutions}

This section is devoted to the proof of the existence of kinetic solutions, as stated in Theorem 6 . According to Theorem 3, this implies the existence of entropy solutions of (1).

Here, the kinetic formulation proves to be very useful: indeed, Eq. (10) is a very simple linear transport equation of which we can compute explicit solutions $f(t, x, v)$ thanks to the method of characteristics. The whole problem is to prove that these solutions can be written in the form $f(t, x, v)=\chi(v, u(t, x))$, possibly up to the addition of the source term $\frac{\partial m}{\partial v}$. With this aim in view, we intend to show that solutions of (10) can be obtained as limits of a BGKtype relaxation model. The steps of the proof are the same as in [7]: first, we study a linear transport equation inspired from Eq. (10). In this first step, the main difference with the homogeneous case lies in the fact that characteristics are no longer straight lines, and the representation formula for solutions of the linear equation is more complicated. Then, we introduce an approached equation for (10), which expresses a relaxation towards an equilibrium of the form $\chi(v, u(t, x))$. There, in order to adapt the proofs of [7] it will be necessary to develop new ideas, especially because Eq. (10) or Eq. (1) are no longer invariant under translations in the variable $x$. Eventually, we prove a few compactness results which allow us to pass to the limit in nonlinear terms as the relaxation parameter goes to $\infty$ (hydrodynamic limit). Once again, these compactness results are tougher to obtain than in the homogeneous case due to the more general form of characteristics which yields rather lengthy calculations.

\subsection{Preliminaries: study of a linear transport equation}

First, let us study the following equation:

$$
\begin{aligned}
& \frac{\partial}{\partial t} f(t, x, v)+\operatorname{div}_{x, v}(a(x, v) f(t, x, v))+\lambda f(t, x, v)=g(t, x, v), \\
& f(t=0, x, v)=f^{0}(x, v)
\end{aligned}
$$

$\lambda>0$ is a fixed parameter, $g$ is a given function which will sometimes be referred to as source term.

Define the characteristic ODE associated to this equation:

$$
\left\{\begin{array}{l}
\dot{X}(t, y, w)=a(X(t, y, w)), \\
X(t=0, y, w)=(y, w) \in \mathbb{R}^{n+1},
\end{array}\right.
$$

$X$ is well defined thanks to hypotheses (5), (6). For all $t \geqslant 0, X(t): \mathbb{R}^{n+1} \rightarrow \mathbb{R}^{n+1}$ is a $\mathcal{C}^{1}$-diffeomorphism and thanks to Liouville's theorem,

$$
J(t, z):=\exp \left(\int_{0}^{t} \operatorname{div}_{x, v} a(X(s, z)) \mathrm{d} s\right)=\left|\operatorname{det} \frac{\partial X(t, z)}{\partial z}\right| .
$$

(Here and in the rest of the section $z$ denotes the couple $(y, w)$ )

Since $\operatorname{div}_{x, v} a=0$, notice that

$$
\left|\operatorname{det} \frac{\partial X(t, z)}{\partial z}\right|=1
$$

We are then able to prove the following

Lemma 10. Let $f^{0} \in L^{1}\left(\mathbb{R}^{n+1}\right), g \in L_{\mathrm{loc}}^{1}\left(\mathbb{R}_{+}, L^{1}\left(\mathbb{R}^{n+1}\right)\right)$; under the assumptions (2), (3), (5), (6), there exists a unique solution $f \in \mathcal{C}\left(\mathbb{R}_{+} ; L^{1}\left(\mathbb{R}^{n+1}\right)\right)$ of Eq. (23) in the sense of distributions, and it has the following properties:

(1) Representation formula: for all $t \geqslant 0$, for a.e. $(y, w) \in \mathbb{R}^{n+1}$,

$$
f(t, X(t, y, w))=f^{0}(y, w) \mathrm{e}^{-\lambda t}+\int_{0}^{t} \mathrm{e}^{-\lambda(t-s)} g(s, X(s, y, w)) \mathrm{d} s
$$


(2) Evolution of the total mass:

$$
\begin{aligned}
& \frac{\mathrm{d}}{\mathrm{d} t} \int f(t, x, v) \mathrm{d} x \mathrm{~d} v+\lambda \int f(t, x, v) \mathrm{d} x \mathrm{~d} v=\int g(t, x, v) \mathrm{d} x \mathrm{~d} v, \\
& \frac{\mathrm{d}}{\mathrm{d} t} \int|f(t, x, v)| \mathrm{d} x \mathrm{~d} v+\lambda \int|f(t, x, v)| \mathrm{d} x \mathrm{~d} v \leqslant \int|g(t, x, v)| \mathrm{d} x \mathrm{~d} v .
\end{aligned}
$$

Proof. The proof of this lemma is left to the reader; it relies on the use of the characteristics: first, the representation formula is shown for classical $\mathcal{C}^{1}$ solutions; the relations (26) and (27) follow from (25) and (24). Then these results are extended to weak solutions in $L^{1}$ by density and passage to the limit. Uniqueness derives from existence of solutions for the dual problem.

\subsection{Relaxation model towards the equilibrium $\chi(v, u)$}

As mentioned previously, we study a BGK-type model which expresses the relaxation of the solutions of a kinetic equation towards an equilibrium of the form $\chi(v, u(t, x))$ :

$$
\left\{\begin{array}{l}
\frac{\partial}{\partial t} f_{\lambda}+\sum_{i=1}^{n} \frac{\partial}{\partial x_{i}}\left(a_{i} f_{\lambda}\right)+\frac{\partial}{\partial v}\left(a_{n+1} f_{\lambda}\right)+\lambda\left[f_{\lambda}-\chi\left(v, u_{\lambda}(t, x)\right)\right]=0 \\
u_{\lambda}(t, x)=\int_{\mathbb{R}} f_{\lambda}(t, x, v) \mathrm{d} v \\
f_{\lambda}(t=0, x, v)=f^{0}(x, v) .
\end{array}\right.
$$

In the rest of this subsection $\lambda$ is a fixed parameter, and therefore we will denote by $f, u$ the functions $f_{\lambda}, u_{\lambda}$.

Sometimes an additional assumption on the initial data $f^{0} \in L^{1}\left(\mathbb{R}^{n+1}\right)$ will be necessary:

$$
\left|f^{0}(x, v)\right|=\operatorname{sgn}(v) f^{0}(x, v) \leqslant 1 .
$$

Proposition 11. Let $f^{0} \in L^{1}\left(\mathbb{R}^{n+1}\right), u^{0}(x):=\int_{\mathbb{R}} f^{0}(x, v) \mathrm{d} v \in L^{1}\left(\mathbb{R}^{n}\right)$.

Under the assumptions (2)-(6), there exists a unique solution $f \in \mathcal{C}\left(\mathbb{R}_{+} ; L^{1}\left(\mathbb{R}^{n+1}\right)\right)$ of the system (28), and $f$ satisfies:

(1) Mass conservation: for all $t \geqslant 0$,

$$
\begin{aligned}
& \int_{\mathbb{R}^{n}} u(t, x) \mathrm{d} x=\int_{\mathbb{R}^{n}} u^{0}(x) \mathrm{d} x, \\
& \|u(t)\|_{L^{1}\left(\mathbb{R}^{n}\right)} \leqslant\|f(t)\|_{L^{1}\left(\mathbb{R}^{n+1}\right)} \leqslant\left\|f^{0}\right\|_{L^{1}\left(\mathbb{R}^{n+1}\right)} ;
\end{aligned}
$$

(2) $L^{1}$ contraction property: for all $t \geqslant 0$, for all solutions $f_{1}, f_{2}$ of (28) with initial data $f_{1}^{0}, f_{2}^{0}$,

$$
\left\|u_{1}(t)-u_{2}(t)\right\|_{L^{1}\left(\mathbb{R}^{n}\right)} \leqslant\left\|f_{1}(t)-f_{2}(t)\right\|_{L^{1}\left(\mathbb{R}^{n+1}\right)} \leqslant\left\|f_{1}^{0}-f_{2}^{0}\right\|_{L^{1}\left(\mathbb{R}^{n+1}\right)} ;
$$

(3) Uniform equicontinuity in space: assume that $f^{0}(x, v)=\chi\left(v, u^{0}(x)\right)$ for some $u^{0} \in L^{1}\left(\mathbb{R}^{n}\right)$. Then for all $T, R>0$, there exists a function $\omega_{R, T}:[0, \infty) \rightarrow[0, \infty)$ independent of $\lambda$, continuous and increasing, with $\omega_{R, T}(0)=0$ such that

$$
\|u(t, \cdot+h)-u(t, \cdot)\|_{L^{1}\left(B_{R}\right)} \leqslant \omega_{R, T}(|h|) \quad \forall t \in[0, T], \forall h \in \mathbb{R}^{n},
$$

where $B_{R}:=\left\{x \in \mathbb{R}^{n} ;|x|<R\right\}$.

(4) Sign property: under the additional hypothesis (29), $f$ satisfies

$$
|f(t, x, v)|=\operatorname{sgn}(v) f(t, x, v) \leqslant 1 ;
$$


(5) $L^{\infty}$ norm control: still assuming that (29) holds, define

$$
A_{\infty}:=\inf \left\{A>0, f^{0}(x, v)=0 \text { for }|v|>A\right\}
$$

then for all $t \geqslant 0$, we have

$$
\begin{aligned}
& \|u(t)\|_{\infty} \leqslant A_{\infty}+t\left\|a_{n+1}\right\|_{\infty}, \\
& f(t, x, v)=0 \text { for }|v|>A_{\infty}+t\left\|a_{n+1}\right\|_{\infty} .
\end{aligned}
$$

Proof. We only give the main steps of the proof. This theorem was first proved by B. Perthame and E. Tadmor in [9] in the homogeneous case. We shall follow the same argument as B. Perthame in [7].

First step: the existence is based on a fixed point method. Fix $T>0$ and define the following Banach spaces:

$$
\begin{aligned}
X_{T}:=\mathcal{C}\left(0, T ; L^{1}\left(\mathbb{R}^{n}\right)\right), & \|u\|_{X}:=\sup _{t \in[0, T]}\|u(t)\|_{L^{1}\left(\mathbb{R}^{n}\right)} ; \\
Y_{T}:=\mathcal{C}\left(0, T ; L^{1}\left(\mathbb{R}^{n+1}\right)\right), & \|f\|_{Y}:=\sup _{t \in[0, T]}\|f(t)\|_{L^{1}\left(\mathbb{R}^{n+1}\right)} .
\end{aligned}
$$

If $w \in X_{T}$, thanks to Lemma 10 , there exists a unique $f \in Y_{T}$ solution of the equation

$$
\left\{\begin{array}{l}
\frac{\partial}{\partial t} f+\operatorname{div}_{x, v}(a f)+\lambda[f-\chi(v, w(t, x))]=0 \\
f(t=0, x, v)=f^{0}(x, v) .
\end{array}\right.
$$

Define the operator $\Phi: w \in X_{T} \mapsto u=\int_{\mathbb{R}} f \mathrm{~d} v \in X_{T}$, where $f \in Y_{T}$ is the solution of (35) corresponding to $w$. Then it is readily proved, using the linearity of Eq. (23), inequality (27) and the property

$$
\int_{\mathbb{R}}\left|\chi\left(v, u_{1}\right)-\chi\left(v, u_{2}\right)\right| \mathrm{d} v=\left|u_{1}-u_{2}\right| \quad \forall u_{1}, u_{2} \in \mathbb{R},
$$

that

$$
\frac{\mathrm{d}}{\mathrm{d} t} \int_{\mathbb{R}^{n+1}}\left|f_{1}-f_{2}\right| \mathrm{d} x \mathrm{~d} v+\lambda \int_{\mathbb{R}^{n+1}}\left|f_{1}-f_{2}\right| \mathrm{d} x \mathrm{~d} v \leqslant \lambda\left\|w_{1}-w_{2}\right\|_{X} .
$$

Hence, by Gronwall's lemma, $\Phi$ is a strict contraction from $X_{T}$ into itself. Thus, $\Phi$ has a unique fixed point in $X_{T}$ for every initial data $f^{0} \in L^{1}\left(\mathbb{R}^{n+1}\right)$, and the function $f$ solution of (35) for the fixed point of $\Phi$ is a solution of (28).

Second step: the proof of (30), (31) and (32) relies on the particular form of the function $\chi$ and on properties (26) and (27) of Lemma 10. It is left to the reader since it does not give rise to any particular difficulty.

Third step: inequality (33) is not so easy to prove since Eq. (23) is not invariant under translations in the $x$ variable; consequently, denoting by $f_{h}(t, x, v)$ the solution corresponding to the translated initial data $f^{0}(x+h, v)$, there is no reason why $f_{h}(t, x, v)$ should be equal to $f(t, x+h, v)$, and thus we cannot apply (32).

Let us estimate $f(t, x, v)-f(t, x+h, v)$, or more generally $f(t, x, v)-f(t, x+h, v+w)$ for all $(h, w) \in \mathbb{R}^{n+1}$; in the sequel, for $k=(h, w) \in \mathbb{R}^{n+1}$ and $\varphi: \mathbb{R}^{n+1} \rightarrow \mathbb{R}^{d}(d \in \mathbb{N}$ arbitrary), we set

$$
\tau_{k} \varphi:(x, v) \in \mathbb{R}^{n+1} \mapsto \varphi(x+h, v+w), \quad J_{k}:=\mathrm{Id}-\tau_{k} .
$$

$f-\tau_{k} f=J_{k} f$ is a solution of

$$
\frac{\partial}{\partial t} J_{k} f+\operatorname{div}_{x, v}\left(\left(\tau_{k} a\right) J_{k} f\right)+\lambda J_{k} f=\lambda J_{k}[\chi(v, u(t, x))]-J_{k} a \cdot \nabla_{x, v} f .
$$

Unfortunately the right-hand side of the above equation does not belong to $L^{1}$. We first derive formally an a priori estimate, under the additional hypothesis $u^{0} \in B V\left(\mathbb{R}^{n}\right) \cap L^{\infty}\left(\mathbb{R}^{n}\right)$, then we will briefly justify rigorously this a priori estimate, and eventually we will generalize our result to the case when $u^{0}$ merely belongs to $L^{1}$.

Let $\alpha>0, T>0$ arbitrary, and let

$$
\varphi_{\alpha}(x):=\exp \left(-\alpha\left(\left|x_{1}\right|+\cdots+\left|x_{n}\right|\right)\right), \quad x \in \mathbb{R}^{n} .
$$

Then $\left|\nabla \varphi_{\alpha}(x)\right| \leqslant \alpha \varphi_{\alpha}(x)$ for almost every $x \in \mathbb{R}^{n}$. 
We denote by $C$ a generic constant which depends only on $n, T, u^{0}$ and the bounds on $a$, but neither on $\alpha$ nor on $\lambda$. We begin with the case when $k=(0, w)$, with $w \in \mathbb{R}$ arbitrary. It is then easily checked that for all $u \in \mathbb{R}$,

$$
\int_{\mathbb{R}}|\chi(v, u)-\chi(v+w, u)| \mathrm{d} v \leqslant 2 \min (|w|,|u|) \quad \forall(w, u) \in \mathbb{R}^{2} .
$$

Thus, multiplying (36) by $\varphi_{\alpha}(x)$ and integrating on $\mathbb{R}^{n+1}$ yields

$$
\begin{aligned}
\frac{\mathrm{d}}{\mathrm{d} t} \int_{\mathbb{R}^{n+1}}\left|J_{k} f\right| \varphi_{\alpha}+\lambda \int_{\mathbb{R}^{n+1}}\left|J_{k} f\right| \varphi_{\alpha} \leqslant & \lambda \frac{C}{\alpha^{n}}|w|+\left\|\partial_{v} a\right\|_{L^{\infty}\left(\mathbb{R}^{n} \times(-A, A)\right)}|w| \int_{\mathbb{R}^{n+1}}\left|\nabla_{x, v} f\right| \varphi_{\alpha}(x) \\
& +\|a\|_{L^{\infty}\left(\mathbb{R}^{n} \times(-A, A)\right)} \int_{\mathbb{R}^{n+1}}\left|J_{k} f\right|\left|\nabla_{x} \varphi_{\alpha}\right| \\
\leqslant & C|w|\left(\frac{\lambda}{\alpha^{n}}+\int_{\mathbb{R}^{n+1}}\left|\nabla_{x, v} f\right| \varphi_{\alpha}(x)\right)+C \alpha \int_{\mathbb{R}^{n+1}}\left|J_{k} f\right| \varphi_{\alpha}
\end{aligned}
$$

where $A=\left\|u^{0}\right\|_{L^{\infty}}+T\left\|a_{n+1}\right\|_{L^{\infty}}$. We have used here the $L^{\infty}$ norm control property, which will be proved later.

We then use Gronwall's lemma, assuming that $\lambda>2$ and $C \alpha<1$, and we obtain

$$
\begin{aligned}
\int_{\mathbb{R}^{n+1}}\left|J_{k} f\right| \varphi_{\alpha} & \leqslant C \mathrm{e}^{-(\lambda-C \alpha) t} \int_{\mathbb{R}^{n+1}}\left|J_{k} f^{0}\right| \varphi_{\alpha}+|w| \frac{C}{\alpha^{n}}+C|w| \int_{0}^{t} \int_{\mathbb{R}^{n+1}} \mathrm{e}^{-(\lambda-C \alpha)(t-s)}\left|\nabla_{x, v} f(s)\right| \varphi_{\alpha} \mathrm{d} s \\
& \leqslant C|w|\left[\frac{1}{\alpha^{n}}+\int_{0}^{t} \int_{\mathbb{R}^{n+1}}\left|\nabla_{x, v} f(s)\right| \varphi_{\alpha} \mathrm{d} s\right] .
\end{aligned}
$$

We now let $w$ go to 0 , which gives

$$
\int_{\mathbb{R}^{n+1}}\left|\nabla_{v} f(t)\right| \varphi_{\alpha} \leqslant C\left[\frac{1}{\alpha^{n}}+\int_{0}^{t} \int_{\mathbb{R}^{n+1}}\left(\left|\nabla_{x} f\right|+\left|\nabla_{v} f\right|\right) \varphi_{\alpha} \mathrm{d} s\right] .
$$

Using Gronwall's lemma for the second time, we derive

$$
\int_{0}^{t} \int_{\mathbb{R}^{n+1}}\left|\nabla_{v} f\right| \varphi_{\alpha} \mathrm{d} s \leqslant C\left[\frac{1}{\alpha^{n}}+\int_{0}^{t} \int_{\mathbb{R}^{n+1}}\left|\nabla_{x} f\right| \varphi_{\alpha} \mathrm{d} s\right] .
$$

Thanks to this bound on $\left|\nabla_{v} f\right|$, we now derive a bound on $\left|\nabla_{x} f\right|$ : take $k=(h, 0)$, with $h \in \mathbb{R}^{n}$ arbitrary. With the same arguments as before, we obtain successively

$$
\begin{gathered}
\frac{\mathrm{d}}{\mathrm{d} t} \int_{\mathbb{R}^{n+1}}\left|J_{k} f\right| \varphi_{\alpha} \leqslant C|h| \int_{\mathbb{R}^{n+1}}\left|\nabla_{x, v} f\right| \varphi_{\alpha}+C \alpha \int_{\mathbb{R}^{n+1}}\left|J_{k} f\right| \varphi_{\alpha}, \\
\int_{\mathbb{R}^{n+1}}\left|J_{k} f\right| \varphi_{\alpha}(t) \leqslant C|h|\left[\left\|\nabla_{x} u^{0}\right\|_{M^{1}}+\int_{0}^{t} \int_{\mathbb{R}^{n+1}}\left|\nabla_{x, v} f\right| \varphi_{\alpha}\right] .
\end{gathered}
$$

We then let $h$ go to 0 , and we use the previous bound on $\left|\nabla_{v} f\right|$; we apply once again Gronwall's lemma, and we are led to

$$
\sup _{t \in[0, T]} \int_{\mathbb{R}^{n+1}}\left|\nabla_{x} f(t)\right| \varphi_{\alpha} \leqslant C\left[\frac{1}{\alpha^{n}}+\left\|\nabla_{x} u^{0}\right\|_{M^{1}}+\int_{0}^{T} \int_{\mathbb{R}^{n+1}}\left|\nabla_{x} f\right| \varphi_{\alpha}\right] \leqslant C\left[\frac{1}{\alpha^{n}}+\left\|\nabla_{x} u^{0}\right\|_{M^{1}}\right] .
$$


Take now $R>0$ arbitrary, $\alpha>0$ small enough so that $\mathrm{e}^{-\alpha R} \geqslant 1 / 2$. Then for all $h \in \mathbb{R}^{n}$, for all $\lambda>2, t \in[0, T]$

$$
\left\|u_{\lambda}(t, \cdot+h)-u_{\lambda}(t, \cdot)\right\|_{L^{1}\left(B_{R}\right)} \leqslant|h| \int_{\mathbb{R}^{n+1}}\left|\nabla_{x} f(t)\right| \varphi_{\alpha} \leqslant C|h|\left[\frac{1}{\alpha^{n}}+\left\|\nabla_{x} u^{0}\right\|_{M^{1}}\right] .
$$

Let us now justify this a priori estimate: we consider a parabolic approximation of the linear transport equation (23)

$$
\partial_{t} f+\operatorname{div}_{x, v}(a f)+\lambda f-\varepsilon \Delta_{x, v} f=g .
$$

The solutions of this equation still satisfy (26), (27). Thus for all $\lambda, \varepsilon>0$ there exists a unique solution of

$$
\partial_{t} f_{\varepsilon, \lambda}+\operatorname{div}_{x, v} f_{\varepsilon, \lambda}+\lambda f_{\varepsilon, \lambda}-\varepsilon \Delta_{x, v} f_{\varepsilon, \lambda}=\lambda \chi\left(v, u_{\varepsilon, \lambda}\right)
$$

with $u_{\varepsilon, \lambda}=\int_{\mathbb{R}} f_{\varepsilon, \lambda}(\cdot, v) \mathrm{d} v$. Moreover, classically $f_{\varepsilon, \lambda} \rightarrow f_{\lambda}$ in $Y_{T}$. And thanks to the regularizing parabolic term $f_{\varepsilon, \lambda}$ has enough regularity for the a priori estimate to be rigorously derived. Passing to the limit as $\varepsilon \rightarrow 0$ in (37) gives the desired result.

Now, when $u^{0}$ does not belong to $L^{\infty} \cap B V\left(\mathbb{R}^{n+1}\right)$, we take a family $u_{\delta}^{0}$ which has these properties and such that $\left\|u^{0}-u_{\delta}^{0}\right\|_{L^{1}\left(\mathbb{R}^{n}\right)} \leqslant \delta$ for all $\delta>0$. Then according to the $L^{1}$ contraction principle and (37) for the solution corresponding to the initial data $\chi\left(v, u_{\delta}^{0}\right)$, we have

$$
\|u(t, \cdot+h)-u(t, \cdot)\|_{L^{1}\left(B_{R}\right)} \leqslant C(\delta, R, T)|h|+2 \delta \quad \forall t \in[0, T] \forall \delta>0 .
$$

Inequality (33) is then proved with

$$
\omega_{R, T}(\eta)=\inf _{\delta>0}[C(\delta, R, T)|h|+2 \delta] .
$$

Fourth step: sign property: if $f^{0}$ satisfies (29), then $h(t, x, v)=\operatorname{sgn}(v) f(t, x, v)$ satisfies Eq. (23) with a source term $g(t, x, v)=\lambda \operatorname{sgn}(v) \chi(v, u(t, x)) \in[0, \lambda]$ and an initial data belonging to $[0,1]$. According to the representation formula $(25), 0 \leqslant h \leqslant 1$ a.e.

Fifth step: $L^{\infty}$ norm control: first, notice that the definition of $A_{\infty}$ only depends on the initial data $f^{0}$. Consider the convex set

$$
\mathcal{C}:=\left\{w \in X_{T} \cap L^{\infty}\left((0, T) \times \mathbb{R}^{n}\right) ;\|w(t)\|_{L^{\infty}\left(\mathbb{R}^{n}\right)} \leqslant A_{\infty}+t\left\|a_{n+1}\right\|_{\infty} \quad \text { for a.e. } t \in(0, T)\right\} .
$$

The idea is to prove that $\mathcal{C}$ is invariant by the application $\Phi$, which entails that the fixed point of $\Phi$ belongs to $\mathcal{C}$. Indeed, the fixed point of $\Phi$ corresponding to the initial data $f^{0}$ is obtained as the limit in $X_{T}$ of any sequence $w_{n}=\Phi^{n}\left(w_{0}\right)$, with $w_{0} \in X_{T}$ arbitrary. Take $w_{0} \in \mathcal{C}$ : if $\Phi$ leaves $\mathcal{C}$ invariant, then $w_{n} \in \mathcal{C}$ for all $n$ and up to a subsequence, $w_{n}$ converges weakly* in $L^{\infty}\left((0, T) \times \mathbb{R}^{n}\right)$. Thus the fixed point of $\Phi$ belongs to $\mathcal{C}$. Here the main difference with the proof of [7] is the use of characteristics and the fact that the $L^{\infty}$ norm is not conserved.

Let us now prove that $\Phi(\mathcal{C}) \subset \mathcal{C}$. Let $w_{1} \in \mathcal{C}, w_{2}=\Phi\left(w_{1}\right)$. Fix $t \in(0, T), x_{0} \in \mathbb{R}^{n}$, and take $v_{0} \in \mathbb{R}$ such that $\left|v_{0}\right|>A_{\infty}+t\left\|a_{n+1}\right\|_{\infty}$; we denote by $(y, w)$ the unique vector in $\mathbb{R}^{n+1}$ such that $X(t, y, w)=\left(x_{0}, v_{0}\right)$, and by $x(s, y, w) \in \mathbb{R}^{n}, v(s, y, w) \in \mathbb{R}$ the functions

$$
X(s, y, w):=(x(s, y, w), v(s, y, w)) \quad \forall s \in[0, T] .
$$

Thus according to hypothesis (3) and to the definition of $X$,

$$
|v(s, y, w)-v(t, y, w)| \leqslant\left\|a_{n+1}\right\|_{\infty}(t-s) \quad \forall s \in[0, T],
$$

which yields

$$
|v(s, y, w)|>A_{\infty}+s\left\|a_{n+1}\right\|_{\infty} \quad \forall s \in[0, t] .
$$

Hence $f^{0}(y, w)=f^{0}(x(0, y, w), v(0, y, w))=0$ and

$$
\chi\left(v(s, y, w), w_{1}(s, x(s, y, w))\right)=0 \quad \text { for } s \in(0, T) .
$$

The representation formula (25) then leads us to

$$
f_{1}\left(t, x_{0}, v_{0}\right)=0 \text { for }\left|v_{0}\right|>A_{\infty}+t\left\|a_{n+1}\right\|_{\infty} .
$$

This equality combined with the sign property (34) entails that $w_{2} \in \mathcal{C}$. 


\subsection{Passage to the limit: compactness of the family $\left(u_{\lambda}, f_{\lambda}\right)$}

The last step of the proof of the existence consists in passing to the limit as $\lambda \rightarrow \infty$ in Eq. (28) (hydrodynamic limit). More precisely we will prove the following proposition:

Proposition 12. Let $u^{0} \in L^{1}\left(\mathbb{R}^{n}\right), f^{0}(x, v):=\chi\left(v, u^{0}(x)\right), T>0$. Assume that (2)-(6) are satisfied.

Let $\left(u_{\lambda}, f_{\lambda}\right)$ be the solution of the system (28) with initial data $f^{0}$. Then as $\lambda \rightarrow \infty$ there exists a subsequence (still denoted by $\lambda$ ) such that for all $T>0$

$$
\begin{aligned}
& u_{\lambda}(t, x) \rightarrow u(t, x) \quad \text { in } \mathcal{C}\left(0, T ; L^{1}\left(\mathbb{R}^{n}\right)\right), \\
& f_{\lambda}(t, x, v) \rightarrow f(t, x, v)=\chi(v, u(t, x)) \quad \text { in } \mathcal{C}\left(0, T ; L^{1}\left(\mathbb{R}^{n+1}\right)\right)
\end{aligned}
$$

and $f(t, x, v)=\chi(v, u)$ is a weak solution of the kinetic equation (10).

Moreover, according to the uniqueness result, the whole family $\left(u_{\lambda}, f_{\lambda}\right)$ converges to $(u, f)$.

Proof. We begin with the compactness of the family $u_{\lambda}$, which follows from Ascoli's theorem.

First step: equicontinuity of the family $u_{\lambda}$.

First, thanks to (33), the family $u_{\lambda}$ is locally equicontinuous in space. Moreover, for all $\lambda>0, t \geqslant 0, k>0$ we have

$$
\left\|u_{\lambda}(t)-u_{\lambda}(t+k)\right\|_{L^{1}\left(\mathbb{R}^{n}\right)} \leqslant\left\|f^{0}-f_{\lambda}(k)\right\|_{L^{1}\left(\mathbb{R}^{n+1}\right)}
$$

thanks to the $L^{1}$ contraction principle.

The difficult part is to prove that $\left\|f^{0}-f_{\lambda}(k)\right\|_{L^{1}\left(\mathbb{R}^{n+1}\right)}$ is bounded uniformly in $\lambda$. Once again we follow the proof of B. Perthame in [7].

In the following, we set

$$
\omega\left(u^{0}, \alpha\right):=\sup _{h \in \mathbb{R}^{n},|h| \leqslant \alpha}\left\|u^{0}(\cdot+h)-u^{0}(\cdot)\right\|_{L^{1}\left(\mathbb{R}^{n}\right)} .
$$

The proof is similar to the one of (33): $f_{\lambda}-f^{0}$ is a solution of

$$
\frac{\partial}{\partial t}\left(f_{\lambda}-f^{0}\right)+\operatorname{div}_{x, v}\left[a\left(f_{\lambda}-f^{0}\right)\right]+\lambda\left(f_{\lambda}-f^{0}\right)=\lambda\left(\chi\left(v, u_{\lambda}\right)-f^{0}\right)-a(x, v) \cdot \nabla_{x, v} f^{0},
$$

but since $f^{0}$ is not regular, we replace $f^{0}$ by $\chi\left(v, u_{\delta}^{0}\right)$ in the above equation, where $u_{\delta}^{0}=u^{0} * \varphi_{\delta}$ and $\varphi_{\delta}=\frac{1}{\delta^{n}} \varphi\left(\frac{\dot{\delta}}{\delta}\right)$, $\varphi$ the standard mollifier. Notice that

$$
\nabla_{x, v} \chi\left(v, u_{\delta}^{0}\right)=\left(\delta\left(v-u_{\delta}^{0}\right) \nabla_{x} u_{\delta}^{0}, \delta(v)-\delta\left(v-u_{\delta}^{0}\right)\right),
$$

and $a_{n+1}(x, v) \delta(v)=0$ in the sense of distributions thanks to (4). Moreover, $u_{\delta}^{0} \in L^{\infty}\left(\mathbb{R}^{n}\right)$ and

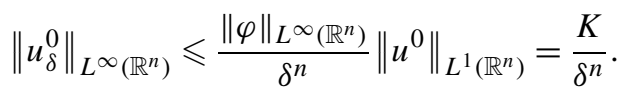

Set

$$
A_{\delta}:=\|a\|_{L^{\infty}\left(\mathbb{R}^{n} \times\left(-K / \delta^{n}, K / \delta^{n}\right)\right)} .
$$

Using property (27) of Lemma 10 yields the following inequality

$$
\frac{\mathrm{d}}{\mathrm{d} t}\left\|f_{\lambda}-\chi\left(v, u_{\delta}^{0}\right)\right\|_{L^{1}\left(\mathbb{R}^{n+1}\right)} \leqslant A_{\delta}\left\|\nabla_{x} \chi\left(v, u_{\delta}^{0}\right)\right\|_{M^{1}\left(\mathbb{R}^{n+1}\right)}+\left\|a_{n+1}\left(\cdot, u_{\delta}^{0}\right)\right\|_{L^{1}\left(\mathbb{R}^{n}\right)}
$$

in which we easily evaluate the different terms

$$
\begin{aligned}
& \left\|\nabla_{x} \chi\left(v, u_{\delta}^{0}\right)\right\|_{M^{1}\left(\mathbb{R}^{n+1}\right)} \leqslant \frac{\|\nabla \varphi\|_{L^{1}\left(\mathbb{R}^{n}\right)}}{\delta} \omega\left(u^{0}, \delta\right), \\
& \left\|f_{\lambda}-\chi\left(v, u^{0}\right)\right\|_{L^{1}\left(\mathbb{R}^{n+1}\right)} \leqslant\left\|f_{\lambda}-\chi\left(v, u_{\delta}^{0}\right)\right\|_{L^{1}\left(\mathbb{R}^{n+1}\right)}+\left\|u^{0}-u_{\delta}^{0}\right\|_{L^{1}\left(\mathbb{R}^{n}\right)}, \\
& \left\|u^{0}-u_{\delta}^{0}\right\|_{L^{1}\left(\mathbb{R}^{n}\right)} \leqslant \int_{\mathbb{R}^{n} \times \mathbb{R}^{n}}\left|u^{0}(x-y)-u^{0}(x)\right| \varphi_{\delta}(y) \mathrm{d} x \mathrm{~d} y \leqslant \omega\left(u^{0}, \delta\right) .
\end{aligned}
$$


Moreover, thanks to hypotheses (4), (5) we also have

$$
\left\|a_{n+1}\left(\cdot, u_{\delta}^{0}\right)\right\|_{L^{1}\left(\mathbb{R}^{n}\right)} \leqslant B_{\delta}\left\|u^{0}\right\|_{L^{1}\left(\mathbb{R}^{n}\right)}, \quad \text { where } B_{\delta}:=\left\|\partial_{v} a_{n+1}\right\|_{L^{\infty}\left(\mathbb{R}^{n} \times\left(-\frac{K}{\delta^{n}}, \frac{K}{\delta^{n}}\right)\right)} .
$$

Eventually we obtain the following bound uniformly in $\lambda>0$

$$
\left\|f_{\lambda}(k)-\chi\left(v, u^{0}\right)\right\|_{L^{1}\left(\mathbb{R}^{n+1}\right)} \leqslant \omega\left(u^{0}, \delta\right)\left[2+k A_{\delta} \frac{\left.\|\nabla \varphi\|_{L^{1}\left(\mathbb{R}^{n}\right)}\right]}{\delta}\right] k B_{\delta}\left\|u^{0}\right\|_{L^{1}\left(\mathbb{R}^{n}\right)} .
$$

We are thus led to define the modulus of equicontinuity in time

$$
\omega^{1}(k):=\inf _{\delta>0}\left\{\omega\left(u^{0}, \delta\right)\left[2+k A_{\delta} \frac{\|\nabla \varphi\|_{L^{1}\left(\mathbb{R}^{n}\right)}}{\delta}\right]+k B_{\delta}\left\|u^{0}\right\|_{L^{1}\left(\mathbb{R}^{n}\right)}\right\} .
$$

Second step: uniform equi-integrability.

The property is first shown in the case of bounded initial data, and then generalized to the case of initial data in $L^{1}\left(\mathbb{R}^{n}\right)$.

If $u^{0} \in L^{\infty}\left(\mathbb{R}^{n}\right)$, according to the $L^{\infty}$ norm control property of Proposition $11, f_{\lambda}(t, x, v)=0$ for a.e. $(t, x) \in$ $\mathbb{R}_{+} \times \mathbb{R}^{n}, v \in \mathbb{R}$ such that $|v|>\left\|u^{0}\right\|_{\infty}+t\left\|a_{n+1}\right\|_{\infty}$. In that case, define

$$
A:=\|a\|_{L^{\infty}\left(\mathbb{R}^{n} \times(-M, M)\right)},
$$

where $M:=\left\|u^{0}\right\|_{\infty}+T\left\|a_{n+1}\right\|_{\infty}$.

Take $\varphi \in \mathcal{C}^{1}\left(\mathbb{R}^{n} ; \mathbb{R}\right)$ such that $\varphi(x)=0$ for $|x| \leqslant \frac{1}{2}, \varphi(x)=1$ for $|x| \geqslant 1$, and $0 \leqslant \varphi \leqslant 1$. Define $\varphi_{R}:=\varphi\left(\frac{\dot{R}}{R}\right)$. Then multiplying (28) by $\varphi_{R}$ yields

$$
\frac{\partial}{\partial t}\left(f_{\lambda} \varphi_{R}\right)+\operatorname{div}_{x, v}\left(a f_{\lambda} \varphi_{R}\right)+\lambda \varphi_{R}\left[f_{\lambda}-\chi\left(v, u_{\lambda}\right)\right]=f_{\lambda} \sum_{i=1}^{n} a_{i} \frac{\partial \varphi_{R}}{\partial x_{i}} .
$$

We deduce from the above inequality that

$$
\frac{\mathrm{d}}{\mathrm{d} t} \int_{\mathbb{R}^{n+1}}\left|f_{\lambda}\right| \varphi_{R} \mathrm{~d} x \mathrm{~d} v \leqslant A \int_{\mathbb{R}^{n+1}}\left|f_{\lambda}\left\|\nabla_{x} \varphi_{R} \mid \mathrm{d} x \mathrm{~d} v \leqslant \frac{A}{R}\right\| \nabla_{x} \varphi\left\|_{\infty}\right\| u^{0} \|_{L^{1}\left(\mathbb{R}^{n}\right)}\right.
$$

which yields eventually

$$
\int_{|x| \geqslant R}\left|u_{\lambda}(t, x)\right| \mathrm{d} x \leqslant \int_{\mathbb{R}^{n+1}}\left|f_{\lambda}(t, x, v)\right| \varphi_{R}(x) \mathrm{d} x \mathrm{~d} v \leqslant \int_{|x| \geqslant R / 2}\left|u^{0}(x)\right| \mathrm{d} x+T \frac{A}{R}\left\|\nabla_{x} \varphi\right\|_{\infty}\left\|u^{0}\right\|_{L^{1}\left(\mathbb{R}^{n}\right)} .
$$

This last inequality shows the uniform equi-integrability of the family $u_{\lambda}$ in the case where $u^{0}$ is bounded.

If $u^{0}$ is not bounded, we regularize $u^{0}$ by convolution: set $u_{\delta}^{0}=u^{0} * \phi_{\delta}$, and let $u_{\lambda, \delta}, f_{\lambda, \delta}$ be the solution of the system (28) with the initial data $\chi\left(v, u_{\delta}^{0}\right)$.

According to the uniform equi-integrability result in the case of bounded initial data, we have, with $A_{\delta}:=$ $\|a\|_{L^{\infty}\left(\mathbb{R}^{n} \times\left(-K / \delta^{n}, K / \delta^{n}\right)\right)}$

$$
\int_{|x| \geqslant R}\left|u_{\lambda, \delta}(t, x)\right| \mathrm{d} x \leqslant \int_{|x| \geqslant R / 2}\left|u_{\delta}^{0}(x)\right| \mathrm{d} x+T \frac{A_{\delta}}{R}\left\|\nabla_{x} \varphi\right\|_{\infty}\left\|u_{\varepsilon}^{0}\right\|_{L^{1}\left(\mathbb{R}^{n}\right)} .
$$

The $L^{1}$ contraction principle (32) yields

$$
\int_{|x| \geqslant R}\left|u_{\lambda}(t, x)\right| \mathrm{d} x \leqslant \int_{|x| \geqslant R / 2}\left|u^{0}(x)\right| \mathrm{d} x+T \frac{A_{\delta}}{R}\left\|\nabla_{x} \varphi\right\|_{\infty}\left\|u^{0}\right\|_{L^{1}\left(\mathbb{R}^{n}\right)}+2\left\|u_{\delta}^{0}-u^{0}\right\|_{L^{1}\left(\mathbb{R}^{n}\right)} .
$$

Choose $\delta>0$ so that the quantity $\left\|u_{\delta}^{0}-u^{0}\right\|_{L^{1}\left(\mathbb{R}^{n}\right)}$ is small enough, and then with that choice of $\delta$, take $R$ sufficiently large so that the two other terms in the right-hand side are small as well. Hence $u_{\lambda}$ is uniformly equi-integrable.

Thus, according to Ascoli's theorem, the family $\left\{u_{\lambda}\right\}_{\lambda>0}$ is compact in $\mathcal{C}\left(0, T ; L^{1}\left(\mathbb{R}^{n}\right)\right)$ for all $T>0$; therefore there exist $u \in \mathcal{C}\left(0, T ; L^{1}\left(\mathbb{R}^{n}\right)\right)$ and a sequence $\lambda(n) \rightarrow \infty$ such that $u_{\lambda(n)}$ converges to $u$ in $\mathcal{C}\left(0, T ; L^{1}\left(\mathbb{R}^{n}\right)\right)$. 
Third step: $f_{\lambda(n)} \rightarrow \chi(v, u)$ in $\mathcal{C}\left(0, T ; L^{1}\left(\mathbb{R}^{n+1}\right)\right)$.

Let us recall the representation formula

$$
f_{\lambda(n)}(t, X(t))=\chi\left(w, u^{0}(y)\right) \mathrm{e}^{-\lambda(n) t}+\lambda(n) \int_{0}^{t} \mathrm{e}^{-\lambda(n)(t-s)} \chi\left(v(s), u_{\lambda(n)}(s, x(s))\right) \mathrm{d} s .
$$

In the above formula, for more simplicity we have omitted the variables $y, w$, which are arguments of the functions $x(s), v(s), X(t)$. We will systematically use this notation in the rest of the proof when no confusion might occur.

Thus

$$
\begin{gathered}
f_{\lambda(n)}(t, X(t))-\chi(v(t), u(t, x(t)))=\left[f^{0}(y, w)-\chi(v(t), u(t, x(t)))\right] \mathrm{e}^{-\lambda(n) t} \\
+\lambda(n) \int_{0}^{t} \mathrm{e}^{-\lambda(n)(t-s)}\left[\chi\left(v(s), u_{\lambda(n)}(s, x(s))\right)-\chi(v(t), u(t, x(t)))\right] \mathrm{d} s .
\end{gathered}
$$

We integrate this equality with respect to $y, w$, recalling (24)

$$
\begin{aligned}
& \left\|f_{\lambda(n)}(t)-\chi(v, u(t))\right\|_{L^{1}\left(\mathbb{R}^{n+1}\right)} \leqslant\left(\left\|f^{0}\right\|_{L^{1}\left(\mathbb{R}^{n+1}\right)}+\|u(t)\|_{L^{1}\left(\mathbb{R}^{n}\right)}\right) \mathrm{e}^{-\lambda(n) t} \\
& \quad+\lambda(n) \int_{0}^{t} \int_{\mathbb{R}^{n+1}} \mathrm{e}^{-\lambda(n)(t-s)}\left|\chi\left(v(s), u_{\lambda(n)}(s, x(s))\right)-\chi(v(t), u(t, x(t)))\right| \mathrm{d} s \mathrm{~d} y \mathrm{~d} w .
\end{aligned}
$$

A few rather lengthy calculations are necessary in order to show that the right-hand side of the inequality converges to 0: first, we write

$$
\begin{aligned}
\mid \chi( & \left.(s), u_{\lambda}(s, x(s))\right)-\chi(v(t), u(t, x(t))) \mid \\
\leqslant & \left|\chi\left(v(s), u_{\lambda}(s, x(s))\right)-\chi(v(s), u(s, x(s)))\right| \\
& +|\chi(v(s), u(s, x(s)))-\chi(v(s), u(s, x(t)))| \\
& +|\chi(v(s), u(s, x(t)))-\chi(v(t), u(s, x(t)))| \\
& +|\chi(v(t), u(s, x(t)))-\chi(v(t), u(t, x(t)))| .
\end{aligned}
$$

We need to bound each of the four integrals generated by the terms of the right-hand side (recall that $x(s), x(t), v(s)$, $v(t)$ are functions of $(y, w))$.

Concerning the integral corresponding to (43), after integrating with respect to $y$ and $w$ and changing the variables from $(y, w)$ to $(x, v)=X(s, y, w)$ (remember that the Jacobian of this change of variables is equal to 1 thanks to (24)), we obtain

$$
\lambda(n) \int_{0}^{t} \int_{\mathbb{R}^{n+1}} \mathrm{e}^{-\lambda(n)(t-s)}\left|\chi\left(v(s), u_{\lambda}(s, x(s))\right)-\chi(v(s), u(s, x(s)))\right| \mathrm{d} s \mathrm{~d} y \mathrm{~d} w \leqslant\left\|u_{\lambda}-u\right\|_{X_{T}} \rightarrow 0 .
$$

The same kind of trick allows us to replace $u$ by any regular function $u_{\delta}$, and the approximation error is then upperbounded by $\left\|u-u_{\delta}\right\|_{X_{T}}$. Therefore when it is necessary we will replace $u$ by a regular approximation of $u$ in $X_{T}$.

In a similar fashion, in the integral corresponding to (46) we use the change of variables $(x, v)=X(t, y, w)$ and we are led to

$$
\lambda(n) \int_{0}^{t} \int_{\mathbb{R}^{n+1}} \mathrm{e}^{-\lambda(n)(t-s)}|\chi(v(t), u(s, x(t)))-\chi(v(t), u(t, x(t)))| \mathrm{d} s \mathrm{~d} y \mathrm{~d} w \leqslant \lambda(n) \int_{0}^{t} \mathrm{e}^{-\lambda(n) s} \omega^{1}(s) \mathrm{d} s \rightarrow 0 .
$$

It remains to bound the integrals corresponding to the two other terms. We begin with (45): set, for $0<s<t$, $(y, w) \in \mathbb{R}^{n+1}$,

$$
h(t, s, y, w):=v(s, y, w)-v(t, y, w)
$$


According to the definition of the flux $X(s),|h(t, s, y, w)| \leqslant\left\|a_{n+1}\right\|_{\infty}(t-s)$.

Define $\tilde{h}(t, s, x, v)=v\left(s, X(t)^{-1}(x, v)\right)-v$ (recall that $X(t): \mathbb{R}^{n+1} \rightarrow \mathbb{R}^{n+1}$ is a $\mathcal{C}^{1}$-diffeomorphism). Then

$$
|\tilde{h}(t, s, x, v)| \leqslant\left\|a_{n+1}\right\|_{\infty}(t-s) .
$$

In the integral corresponding to (45) we change the variables from $(y, w)$ to $(x, v)=X(t, y, w)$ :

$$
\begin{aligned}
& \lambda(n) \int_{0}^{t} \int_{\mathbb{R}^{n+1}} \mathrm{e}^{-\lambda(n)(t-s)}|\chi(v(s), u(s, x(t)))-\chi(v(t), u(s, x(t)))|(y, w) \mathrm{d} s \mathrm{~d} y \mathrm{~d} w \\
& =\lambda(n) \int_{0}^{t} \int_{\mathbb{R}^{n+1}} \mathrm{e}^{-\lambda(n)(t-s)}|\chi(v+\tilde{h}(t, s, x, v), u(s, x))-\chi(v, u(s, x))| \mathrm{d} s \mathrm{~d} x \mathrm{~d} v \\
& \leqslant \lambda(n) \int_{0}^{t} \int_{\mathbb{R}^{n+1}} \mathrm{e}^{-\lambda(n)(t-s)}|\chi(v+|\tilde{h}(t, s, x, v)|, u(s, x))-\chi(v, u(s, x))| \mathrm{d} s \mathrm{~d} x \mathrm{~d} v \\
& \quad+\lambda(n) \int_{0}^{t} \int_{\mathbb{R}^{n+1}} \mathrm{e}^{-\lambda(n)(t-s)}|\chi(v-|\tilde{h}(t, s, x, v)|, u(s, x))-\chi(v, u(s, x))| \mathrm{d} s \mathrm{~d} x \mathrm{~d} v .
\end{aligned}
$$

Let us upper-bound for instance $|\chi(v+|\tilde{h}|, u)-\chi(v, u)|$ :

$$
\begin{aligned}
& |\chi(v+|\tilde{h}(t, s, x, v)|, u(s, x))-\chi(v, u(s, x))| \leqslant\left|\chi\left(v+\left\|a_{n+1}\right\|_{\infty}(t-s), u(s, x)\right)-\chi(v, u(s, x))\right| \\
& \quad+\mathbf{1}_{u(s, x)<0} \mathbf{1}_{-\left\|a_{n+1}\right\|_{\infty}(t-s)<v<u(s, x)}+\mathbf{1}_{u(s, x)>0} \mathbf{1}_{u(s, x)-\left\|a_{n+1}\right\|_{\infty}(t-s)<v<0 .}
\end{aligned}
$$

Integrating this inequality on $\mathbb{R}^{n+1}$ yields

$$
\begin{aligned}
& \iint_{\mathbb{R}^{n}} \mathrm{e}_{\mathbb{R}} \\
& \quad \leqslant\left\|\inf \left(|u(s)|,\left\|a_{n+1}\right\|_{\infty}(t-s)\right)\right\|_{L^{1}\left(\mathbb{R}^{n}\right)}+\left\|\mathbf{1}_{u(s)<0}\left(u+\left\|a_{n+1}\right\|_{\infty}(t-s)\right)_{+}\right\|_{L^{1}\left(\mathbb{R}^{n}\right)} \\
& \quad+\left\|\mathbf{1}_{u(s)>0}\left(u-\left\|a_{n+1}\right\|_{\infty}(t-s)\right)_{-}\right\|_{L^{1}\left(\mathbb{R}^{n}\right)} .
\end{aligned}
$$

Each of the terms in the right-hand side goes to 0 as $s \rightarrow t$. Therefore

$$
\lambda(n) \int_{0}^{t} \int_{\mathbb{R}^{n}} \int_{\mathbb{R}} \mathrm{e}^{-\lambda(n)(t-s)}|\chi(v+|\tilde{h}(t, s, x, v)|, u(s, x))-\chi(v, u(s, x))| \mathrm{d} s \mathrm{~d} x \mathrm{~d} v \underset{n \rightarrow \infty}{\longrightarrow} 0 .
$$

The integral corresponding to $|\chi(v-|\tilde{h}|, u)-\chi(v, u)|$ can be treated in a similar fashion.

We now derive a bound for (44); the main difficulty here comes from the lack of regularity of $u$. Thus we consider a function $u_{\delta} \in \mathcal{C}^{1}\left([0, T] \times \mathbb{R}^{n}\right)$ such that $\operatorname{Supp} u_{\delta}(t) \subset K_{\delta} \forall t \in[0, T]$, with $K_{\delta}$ a compact set of $\mathbb{R}^{n}$, and $\| u_{\delta}(t)-$ $u(t) \|_{L^{1}\left(\mathbb{R}^{n}\right)} \leqslant \delta$ for all $t \in[0, T]$. As previously observed, replacing $u$ by $u_{\delta}$ in the integral corresponding to (44) and integrating with respect to $y, w$ leads to an approximation error which can be upper-bounded by $\left\|u-u_{\delta}\right\|_{X_{T}} \leqslant \delta$.

Let us focus on the integral

$$
\int_{\mathbb{R}^{n+1}}\left|\chi\left(v(s, y, w), u_{\delta}(s, x(s, y, w))\right)-\chi\left(v(s, y, w), u_{\delta}(s, x(t, y, w))\right)\right| \mathrm{d} y \mathrm{~d} w .
$$

In order to derive an upper bound for (47), we use the same kind of method as for (45). Set $H(s, t, y, w)=x(t, y, w)-$ $x(s, y, w)$. Then for $0<s<t$ and $1 \leqslant i \leqslant n$, we have

$$
H_{i}(s, t, y, w)=\int_{s}^{t} a_{i}(x(r, y, w), v(r, y, w)) \mathrm{d} r .
$$


Assume that $|v(s, y, w)| \leqslant\left\|u_{\delta}\right\|_{\infty}$; then

$$
|v(r, y, w)| \leqslant\left\|u_{\delta}\right\|_{\infty}+(r-s)\left\|a_{n+1}\right\|_{\infty} \leqslant\left\|u_{\delta}\right\|_{\infty}+T\left\|a_{n+1}\right\|_{\infty} \quad \forall s<r<t .
$$

Therefore there exists $A_{\delta}>0$ such that if $|v(s, y, v)| \leqslant\left\|u_{\delta}\right\|_{\infty}$, then

$$
|H(s, t, y, w)| \leqslant A_{\delta}(t-s) .
$$

Now define $\widetilde{H}(s, t, x, v)=H\left(s, t, X(s)^{-1}(x, v)\right)$ (recall that $X(s)$ is a $\mathcal{C}^{1}$ diffeomorphism of $\left.\mathbb{R}^{n+1}\right)$. We have just proved that there exists $A_{\delta}>0$ such that

$$
|v| \leqslant\left\|u_{\delta}\right\|_{\infty} \Rightarrow|\widetilde{H}(s, t, x, v)| \leqslant A_{\delta}(t-s) .
$$

We are now ready to upper-bound (47):

$$
\begin{aligned}
& \int_{\mathbb{R}^{n+1}}\left|\chi\left(v(s, y, w), u_{\delta}(s, x(s, y, w))\right)-\chi\left(v(s, y, w), u_{\delta}(s, x(t, y, w))\right)\right| \mathrm{d} y \mathrm{~d} w \\
& \quad=\int_{\mathbb{R}^{n+1}}\left|\chi\left(v, u_{\delta}(s, x)\right)-\chi\left(v, u_{\delta}(s, x+\widetilde{H}(s, t, x, v))\right)\right| \mathrm{d} x \mathrm{~d} v \\
& =\int_{\mathbb{R}^{n}} \int_{|v| \leqslant\left\|u_{\delta}\right\|_{\infty}} \mathbf{1}_{v \in\left[u_{\delta}(s, x), u_{\delta}(s, x+\widetilde{H}(s, t, x, v))\right]} \mathrm{d} x \mathrm{~d} v \\
& \leqslant \int_{\mathbb{R}^{n}} \int_{\mathbb{R}} \mathbf{1}_{I_{\delta}(t, s, x)}(v) \mathrm{d} v \mathrm{~d} x \quad \text { where } I_{\delta}(t, s, x)=\bigcup_{h \in \mathbb{R}^{n},}\left[u_{\delta}(t, x), u_{\delta}(t, x+h)\right] \\
& \leqslant \int_{\mathbb{R}^{n}(t-s)} \sup _{\substack{h \in \mathbb{R}^{n},|h| \leqslant A_{\delta}(t-s)}}\left|u_{\delta}(t, x)-u_{\delta}(t, x+h)\right| \mathrm{d} x \leqslant \int_{K_{\delta}+B\left(0, A_{\delta} t\right)}\left\|\nabla u_{\delta}\right\|_{\infty} A_{\delta}(t-s) \mathrm{d} x \\
& \leqslant A_{\delta}(t-s) \operatorname{mes}\left(K_{\delta}+B\left(0, A_{\delta} t\right)\right)\left\|\nabla u_{\delta}\right\|_{\infty} .
\end{aligned}
$$

Hence

$$
\begin{aligned}
& \lambda(n) \int_{0}^{t} \int_{\mathbb{R}^{n+1}} \mathrm{e}^{-\lambda(n)(t-s)}\left|\chi\left(v(s), u_{\delta}(s, x(s))\right)-\chi\left(v(s), u_{\delta}(s, x(t))\right)\right|(y, w) \mathrm{d} y \mathrm{~d} w \\
& \quad \leqslant \frac{1}{\lambda(n)} A_{\delta} \operatorname{mes}\left(\operatorname{Supp} u_{\delta}+B\left(0, A_{\delta} t\right)\right)\left\|\nabla u_{\delta}\right\|_{\infty}
\end{aligned}
$$

(44) is thus upper-bounded by

$$
\inf _{\delta>0}\left[\delta+\frac{1}{\lambda(n)} A_{\delta} \operatorname{mes}\left(\operatorname{Supp} u_{\delta}+B\left(0, A_{\delta} t\right)\right)\left\|\nabla u_{\delta}\right\|_{\infty}\right] \underset{n \rightarrow \infty}{\longrightarrow} 0 .
$$

Thus we have showed that for all $T>0$, there exists $u \in \mathcal{C}\left(0, T ; L^{1}\left(\mathbb{R}^{n}\right)\right)$ and a sequence $\lambda(n) \rightarrow \infty$ such that

$$
\begin{aligned}
& u_{\lambda(n)} \rightarrow u \quad \text { in } \mathcal{C}\left(0, T ; L^{1}\left(\mathbb{R}^{n}\right)\right), \\
& f_{\lambda(n)} \rightarrow f=\chi(\cdot, u) \quad \text { in } \mathcal{C}\left(0, T ; L^{1}\left(\mathbb{R}^{n+1}\right)\right) .
\end{aligned}
$$

Fourth step: $\chi(v, u)$ is a solution of $(10)$.

First, let us recall the equation satisfied by $f_{\lambda}$.

$$
\left\{\begin{array}{l}
\frac{\partial}{\partial t} f_{\lambda}+\operatorname{div}_{x, v}\left(a f_{\lambda}\right)=\lambda\left[\chi\left(v, u_{\lambda}(t, x)\right)-f_{\lambda}\right] \\
u_{\lambda}(t, x)=\int_{\mathbb{R}} f_{\lambda}(t, x, v) \mathrm{d} v \\
f_{\lambda}(t=0, x, v)=f^{0}(x, v)=\chi\left(v, u^{0}(x)\right) .
\end{array}\right.
$$


The following lemma allows us to transform the right-hand side of the evolution equation satisfied by $f_{\lambda}$ :

Lemma 13. Let $g \in L^{1}(\mathbb{R})$ such that

$$
\operatorname{sgn}(v) g(v)=|g(v)| \leqslant 1 \quad \text { a.e. }
$$

Let $w=\int_{\mathbb{R}} g(v) \mathrm{d} v$. There exists a unique function $m \in \mathcal{C}_{0}(\mathbb{R})$ such that

$$
\chi(v, w)-g(v)=\frac{\mathrm{d}}{\mathrm{d} v} m(v) .
$$

Moreover, $m$ is nonnegative.

(A proof of this lemma is given in [7].)

Therefore $f_{\lambda}$ satisfies a kinetic equation of the kind

$$
\frac{\partial}{\partial t} f_{\lambda}+\operatorname{div}_{x, v}\left(a f_{\lambda}\right)=\frac{\partial}{\partial v} m_{\lambda}(t, x, v)
$$

where $m_{\lambda}$ is a nonnegative measure and $m_{\lambda}(t, x, \cdot) \in \mathcal{C}_{0}(\mathbb{R})$ for almost every $(t, x)$.

In order to pass to the limit in the above equation, we need to prove that the family $m_{\lambda}$ is bounded uniformly in $\lambda$; precisely, $m_{\lambda}$ satisfies the following inequality for a.e. $v \in \mathbb{R}$, for all $T>0$

$$
\int_{0}^{T} \int_{\mathbb{R}^{n}} m_{\lambda}(t, x, v) \mathrm{d} x \mathrm{~d} t \leqslant \mu(v)+\int_{0}^{T} \int_{\mathbb{R}^{n}} a_{n+1}(x, v) f_{\lambda}(t, x, v) \mathrm{d} x \mathrm{~d} t
$$

where

$$
\mu(v):=\mathbf{1}_{v \geqslant 0}\left\|\left(u^{0}-v\right)_{+}\right\|_{L^{1}\left(\mathbb{R}^{n}\right)}+\mathbf{1}_{v \geqslant 0}\left\|\left(u^{0}-v\right)_{-}\right\|_{L^{1}\left(\mathbb{R}^{n}\right)} \leqslant\left\|u^{0}\right\|_{L^{1}\left(\mathbb{R}^{n}\right)} .
$$

The proof of this inequality is similar to the one of the bound on $m$ at the end of Theorem 3. First, we prove that for all functions $S \in \mathcal{C}^{2}(\mathbb{R})$ such that $S(0)=0$ and $S^{\prime}$ has bounded support we have

$$
\begin{aligned}
& \int_{0}^{T} \int_{\mathbb{R}^{n+1}} S^{\prime \prime}(v) m_{\lambda}(t, x, v) \mathrm{d} x \mathrm{~d} v \mathrm{~d} t \\
& \quad \leqslant \int_{\mathbb{R}^{n+1}} S^{\prime}(v)\left(\chi\left(v, u^{0}(x)\right)-f_{\lambda}(T, x, v)\right) \mathrm{d} v \mathrm{~d} x+\int_{0}^{T} \int_{\mathbb{R}^{n+1}} a_{n+1}(x, v) f_{\lambda}(t, x, v) S^{\prime \prime}(v) \mathrm{d} v \mathrm{~d} x \mathrm{~d} t .
\end{aligned}
$$

Then we generalize this result to functions of the type $S(v)=\left(v-v_{0}\right)_{+}\left(v_{0}>0\right)$ and $S(v)=\left(v-v_{0}\right)_{-}\left(v_{0}<0\right)$ thanks to regularizations, which leads to (49).

Integrating (49) with respect to $v$ on the interval $(-R, R)$ (with $R>0$ arbitrary) yields

$$
\iint_{0}^{T} \int_{\mathbb{R}^{n}}^{R} m_{\lambda}(t, x, v) \mathrm{d} v \mathrm{~d} x \mathrm{~d} t \leqslant 2 R\left\|u^{0}\right\|_{L^{1}\left(\mathbb{R}^{n}\right)}+T\left\|a_{n+1}\right\|_{L^{\infty}\left(\mathbb{R}^{n+1}\right)}\left\|u^{0}\right\|_{L^{1}\left(\mathbb{R}^{n}\right)} ;
$$

thus we can extract a sub-sequence, still denoted by $\lambda(n)$, such that $m_{\lambda(n)}$ converges in $w-M^{1}$ to a nonnegative measure $m$ bounded on $(0, T) \times \mathbb{R}^{n} \times(-R, R)$ for $R>0$ arbitrary, and which satisfies inequalities of the type (50). Taking $S(v)=\frac{v^{2}}{2}$ leads to the fact that $m$ is bounded on $(0, T) \times \mathbb{R}^{n+1}$ if $u^{0} \in L^{2}$. Eventually, passing to the limit in (48) yields

$$
\frac{\partial}{\partial t} \chi(v, u(t, x))+\operatorname{div}_{x, v}(a(x, v) \chi(v, u(t, x)))=\frac{\partial}{\partial v} m(t, x, v)
$$

and thus $u$ is an entropy solution of (1).

Moreover, it follows from the uniqueness result that the whole families $f_{\lambda}$ and $m_{\lambda}$ converge to $\chi(v, u(t, x))$ and $u$ in $\mathcal{C}\left(0, T ; L^{1}\left(R^{n+1}\right)\right)$ and $\mathcal{C}\left(0, T ; L^{1}\left(R^{n}\right)\right)$ respectively, and $u$ is then well defined on $[0,+\infty) \times \mathbb{R}^{n}$. 


\section{Uniqueness $-L^{1}$ contraction principle}

This section is devoted to the proof of the uniqueness of kinetic (and thus entropy) solutions of (1), in a general $L^{1}$ setting. The classical proof by Kruzkhov of the $L^{1}$ contraction principle (see [3,4] in the general case, and also [10] in the case of a one-dimensional homogeneous conservation law) relies on the use of the so-called Kruzkhov entropies $S(u)=|u-k|$ and on the doubling of the variables. It requires to work with entropy solutions in $\mathcal{C}\left(\mathbb{R}_{+}, L^{1}\right) \cap$ $L_{\text {loc }}^{\infty}\left(\mathbb{R}_{+}, L^{\infty}\left(\mathbb{R}^{n}\right)\right.$ ). Here, we will follow the proof of B. Perthame in [7,8] (see also [2] for another formulation of the same result using measure valued solutions): uniqueness is in fact proved in a broader class of solutions, called generalized kinetic solutions. Some parts of the proof are rather technical, since they involve regularization by convolution of Eq. (10) and strong convergence results in order to pass to the limit in nonlinear terms. We will pass quickly on the details which are treated in [7] and [8] in order to focus on the difficulties which did not occur in the homogeneous case.

Let us start with a definition:

Definition 14. A function $f \in L_{\mathrm{loc}}^{\infty}\left(0,+\infty ; L^{1}\left(\mathbb{R}^{n+1}\right)\right)$ is called a generalized kinetic solution of the scalar conservation law (1) if there exists a nonnegative measure $m$ such that $(f, m)$ is a distributional solution of the system

$$
\begin{aligned}
& \partial_{t} f(t, x, v)+\operatorname{div}_{x, v}(a(x, v) f(t, x, v))=\partial_{v} m(t, x, v), \\
& f(t=0, x, v)=\chi\left(v, u^{0}(x)\right), \quad u^{0} \in L^{1}\left(\mathbb{R}^{n}\right),
\end{aligned}
$$

and if there exists a family $\mu_{T} \in L_{0}^{\infty}(\mathbb{R}), T>0$ and a nonnegative measure $v$ such that

$$
\begin{aligned}
& \iint_{0}^{T} m(t, x, v) \mathrm{d} x \mathrm{~d} t \leqslant \mu_{T}(v) \quad \text { a.e. } v \in \mathbb{R} \forall T>0, \\
& |f(t, x, v)|=\operatorname{sgn}(v) f(t, x, v) \leqslant 1, \\
& \frac{\partial f}{\partial v}=\delta(v)-v(t, x, v) .
\end{aligned}
$$

Remark 15. Without any loss of generality, we assume in the following that the family $\mu_{T}$ is increasing in $T$, and that $\mu_{T}$ nonincreasing (resp. nondecreasing) on $(0,+\infty)$ (resp. $(-\infty, 0)$ ) for each $T>0$. Recall that $L_{0}^{\infty}(\mathbb{R})$ is the subset of $L^{\infty}(\mathbb{R})$ which consists of functions which go to 0 at $\pm \infty$.

Remark 16. It is easily checked that if $u$ is a kinetic solution of $(1)$, then $\chi(v, u)$ is a generalized kinetic solution of (1) with $m$ the kinetic entropy defect measure. In that case $v(t, x, v)=\delta(v-u(t, x))$ and using inequality (19) with $S(v)=\left(v-v_{0}\right)_{+}\left(\right.$resp. $\left.S(v)=\left(v-v_{0}\right)_{-}\right)$with $v_{0}>0$ (resp. $\left.v_{0}<0\right)$ yields inequality (52) with

$$
\begin{aligned}
\mu_{T}(v):= & \mathbf{1}_{v \geqslant 0}\left\|\left(u^{0}-v\right)_{+}\right\|_{L^{1}\left(\mathbb{R}^{n}\right)}+\mathbf{1}_{v \leqslant 0}\left\|\left(u^{0}-v\right)_{-}\right\|_{L^{1}\left(\mathbb{R}^{n}\right)} \\
& +\left\|a_{n+1}\right\|_{\infty} \operatorname{mes}\left\{(t, x) \in(0, T) \times \mathbb{R}^{n},|u(t, x)|>|v|\right\} .
\end{aligned}
$$

Notice also that condition (54) implies that $f$ is monotonous (in $v$, for almost every $(t, x)$ ) on each of the intervals $(0,+\infty),(-\infty, 0)$, and since $f \in L^{1}$,

$$
f(t, x, v) \rightarrow 0 \quad \text { as }|v| \rightarrow \infty
$$

almost everywhere in $(t, x)$. Consequently, integrating now (54) between $-\infty$ and $+\infty$ yields

$$
\int_{\mathbb{R}} v(t, x, v) \mathrm{d} v=1 \text { for a.e. } t, x .
$$

For more simplicity, we will also assume that $f$ is continuous at $t=0$ with values in $L^{1}\left(\mathbb{R}^{n+1}\right)$. This property can in fact be derived from Eq. (51) (see [11,7]); it is always satisfied for entropy solutions.

The result we will prove here is the following: 
Theorem 17. Assume that (2)-(4), (7) are satisfied.

Let $u^{0} \in L^{1}\left(\mathbb{R}^{n}\right)$, and let $f$ be a generalized kinetic solution of the scalar conservation law (1) which is continuous at $t=0$ and such that

$$
\int_{0}^{T} \int_{\mathbb{R}^{n+1}}\left|a_{i}(x, v)\right||f(t, x, v)| \mathrm{d} t \mathrm{~d} x \mathrm{~d} v<+\infty \quad \forall T>0(1 \leqslant i \leqslant n) .
$$

Then there exists $u \in L_{\mathrm{loc}}^{\infty}\left(0,+\infty ; L^{1}\left(\mathbb{R}^{n}\right)\right)$ such that $f(t, x, v)=\chi(v, u(t, x))$ almost everywhere, where $u$ is a solution of (1) (in the sense of distributions).

Moreover, let $f_{1}=\chi\left(v, u_{1}\right), f_{2}=\chi\left(v, u_{2}\right)$ be two generalized kinetic solutions of (1) with initial data $\chi\left(v, u_{1}^{0}\right)$, $\chi\left(v, u_{2}^{0}\right)$. Assume that $f_{j}$ satisfies (56) for $j=1,2$, and that $f_{j}$ is continuous at time $t=0$. Then the $L^{1}$ contraction principle holds: for almost every $t>0$,

$$
\int_{\mathbb{R}^{n}}\left|u_{1}(t, x)-u_{2}(t, x)\right| \mathrm{d} x=\int_{\mathbb{R}^{n+1}}\left|f_{1}-f_{2}\right|(t, x, v) \mathrm{d} x \mathrm{~d} v \leqslant \int_{\mathbb{R}^{n}}\left|u_{1}^{0}(x)-u_{2}^{0}\right| \mathrm{d} x .
$$

The first step of the proof is the regularization by convolution of Eq. (51) in order to use the chain rule for generalized kinetic solutions and compare $f^{2}$ and $|f|$. Take three mollifiers $\varphi_{1} \in \mathcal{D}(\mathbb{R}), \varphi_{2} \in \mathcal{D}\left(\mathbb{R}^{n}\right), \varphi_{3} \in \mathcal{D}(\mathbb{R}), \varphi_{i} \geqslant 0$ $(i=1,2,3)$, with

$$
\int_{\mathbb{R}} \varphi_{1}=\int_{\mathbb{R}^{n}} \varphi_{2}=\int_{\mathbb{R}} \varphi_{3}=1 .
$$

Assume that $\operatorname{supp} \varphi_{1} \subset[-1,0], \operatorname{supp} \varphi_{2} \subset B(0,1), \operatorname{supp} \varphi_{3} \subset[-1,1]$.

For $\varepsilon>0$, set

$$
\varphi_{\varepsilon}(t, x, v):=\frac{1}{\varepsilon^{n+2}} \varphi_{1}\left(\frac{t}{\varepsilon}\right) \varphi_{2}\left(\frac{x}{\varepsilon}\right) \varphi_{3}\left(\frac{v}{\varepsilon}\right), \quad t \in \mathbb{R}, x \in \mathbb{R}^{n}, v \in \mathbb{R} .
$$

Define, for any couple $(f, m)$ solution of $(51)$

$$
f_{\varepsilon}:=f * \varphi_{\varepsilon}, \quad m_{\varepsilon}:=m * \varphi_{\varepsilon} .
$$

Lemma 18. $f_{\varepsilon}$ satisfies the following equation in $\mathcal{D}^{\prime}\left(\mathbb{R}^{n+1}\right)$

$$
\frac{\partial}{\partial t} f_{\varepsilon}+\operatorname{div}_{x, v}\left(a f_{\varepsilon}\right)=\frac{\partial}{\partial v} m_{\varepsilon}+r_{\varepsilon},
$$

where the remainder $r_{\varepsilon}$ goes to 0 in $L_{\mathrm{loc}}^{1}\left((0, \infty) \times \mathbb{R}^{n+1}\right)$ as $\varepsilon \rightarrow 0$ for all $T>0$.

Moreover, for all $\phi \in \mathcal{D}\left([0, \infty) \times \mathbb{R}^{n}\right)$, the following inequality holds

$$
0 \leqslant\left|\int_{0}^{T} \int_{\mathbb{R}^{n}} m_{\varepsilon}(t, x, v) \phi(t, x) \mathrm{d} t \mathrm{~d} x\right| \leqslant\|\phi\|_{\infty} \sup \left(\mu_{T+\varepsilon}(v-\varepsilon), \mu_{T+\varepsilon}(v+\varepsilon)\right) .
$$

Proof. Since

$$
r_{\varepsilon}(t, x, v)=\operatorname{div}_{x, v}\left(a f_{\varepsilon}\right)-\operatorname{div}_{x, v}\left((a f) * \varphi_{\varepsilon}\right),
$$

$r_{\varepsilon}$ can be written as

$$
\begin{aligned}
r_{\varepsilon}(t, z) & =\sum_{i=1}^{n+1} \int_{0}^{\infty} \int_{\mathbb{R}^{n+1}}\left[a_{i}\left(z-z^{\prime}\right)-a_{i}(z)\right] f\left(t-s, z-z^{\prime}\right) \partial_{i} \varphi_{\varepsilon}\left(s, z^{\prime}\right) \mathrm{d} z^{\prime} \mathrm{d} s \\
& =\sum_{1 \leqslant i, j \leqslant n+1} \iint_{0}^{1} \int_{0}^{\infty}\left[\partial_{j} a_{i}(z)-\partial_{j} a_{i}\left(z-\tau z^{\prime}\right)\right] f\left(t-s, z-z^{\prime}\right) z_{j} \partial_{i} \varphi_{\varepsilon}\left(s, z^{\prime}\right) \mathrm{d} z^{\prime} \mathrm{d} s \mathrm{~d} \tau
\end{aligned}
$$




$$
+\sum_{1 \leqslant i, j \leqslant n+1} \partial_{j} a_{i}(z) \int_{0}^{\infty} \int_{\mathbb{R}^{n+1}} f\left(t-s, z-z^{\prime}\right) z_{j} \partial_{i} \varphi_{\varepsilon}\left(s, z^{\prime}\right) \mathrm{d} z^{\prime} \mathrm{d} s .
$$

(In the above inequalities, we have used the notations $z=(x, v) \in \mathbb{R}^{n+1}$ and $\partial_{i}=\partial_{x_{i}}$ for $1 \leqslant i \leqslant n, \partial_{n+1}=\partial_{v}$.)

Since $\operatorname{div}_{x, v} a=0$, the last term in the right-hand side goes to 0 in $L_{\mathrm{loc}}^{1}\left((0, \infty) \times \mathbb{R}^{n+1}\right)$ as $\varepsilon \rightarrow 0$. As for the other term, for any $R, R^{\prime}>0$,

$$
\begin{aligned}
& \int_{|x| \leqslant R,|v| \leqslant R^{\prime}} \mathrm{d} z \int_{0}^{1} \int_{0}^{\infty} \int_{\mathbb{R}^{n+1}}\left|\partial_{j} a_{i}(z)-\partial_{j} a_{i}\left(z-\tau z^{\prime}\right) \| f\left(t-s, z-z^{\prime}\right)\right|\left|z_{j} \partial_{i} \varphi_{\varepsilon}\left(s, z^{\prime}\right)\right| \mathrm{d} z^{\prime} \mathrm{d} s \mathrm{~d} \tau \\
& \leqslant\left\|z_{j} \partial_{i} \varphi\right\|_{L^{1}\left((0, \infty) \times \mathbb{R}^{n+1}\right)} \omega_{R, R^{\prime}}\left(\partial_{j} a_{i}, \varepsilon\right),
\end{aligned}
$$

where

$$
\omega_{R, R^{\prime}}(f, h):=\sup _{|z| \leqslant h}\|f(\cdot+z)-f(\cdot)\|_{L^{1}\left(B_{R} \times\left(-R^{\prime}, R^{\prime}\right)\right)}
$$

for any function $f \in L_{\text {loc }}^{1}\left(\mathbb{R}^{n+1}\right)$.

Thus $r_{\varepsilon} \rightarrow 0$ in $L_{\text {loc }}^{1}\left((0, \infty) \times \mathbb{R}^{n+1}\right)$ as $\varepsilon \rightarrow 0$ for all $T>0$.

The bound on $m_{\varepsilon}$ is easily proved with the assumptions on the function $\mu_{T}$ and is left to the reader.

In a second step, we prove the following

Proposition 19. Let $f$ be a generalized kinetic solution of Eq. (1) continuous at time $t=0$ and satisfying (56). Then there exists $u \in L_{\mathrm{loc}}^{\infty}\left((0,+\infty) ; L^{1}\left(\mathbb{R}^{n}\right)\right)$ such that $f(t, x, v)=\chi(v, u(t, x))$ almost everywhere.

Moreover, for all nonnegative test function $\psi \in \mathcal{D}\left(\mathbb{R}^{n+1}\right)$, for all $T>0$,

$$
\int_{0}^{T} \int_{\mathbb{R}^{n+1}} m_{\varepsilon}(t, x, v) v * \varphi_{\varepsilon}(t, x, v) \psi(x, v) \mathrm{d} t \mathrm{~d} x \mathrm{~d} v \rightarrow 0 \quad \text { as } \varepsilon \rightarrow 0
$$

where $v(t, x, v)=\delta(v-u(t, x))$.

Proof. The proof relies on the comparison between $f^{2}$ and $|f|=\operatorname{sgn}(v) f$. First, let us define

$$
\operatorname{sgn}_{\varepsilon}(v):=\operatorname{sgn} * \varphi_{\varepsilon}(v) ;
$$

then the function $g_{\varepsilon}(t, x, v):=\operatorname{sgn}_{\varepsilon}(v) f_{\varepsilon}(t, x, v)$ satisfies

$$
\partial_{t} g_{\varepsilon}+\operatorname{div}_{x, v}\left(a g_{\varepsilon}\right)=\frac{\partial m_{\varepsilon}}{\partial v} \operatorname{sgn}_{\varepsilon}(v)+r_{\varepsilon} \operatorname{sgn}_{\varepsilon}(v)+\operatorname{sgn}_{\varepsilon}^{\prime}(v) a_{n+1}(x, v) f_{\varepsilon} ;
$$

On the other hand, $f_{\varepsilon}^{2}$ satisfies

$$
\partial_{t} f_{\varepsilon}^{2}+\operatorname{div}_{x, v}\left(a f_{\varepsilon}^{2}\right)=2 \frac{\partial m_{\varepsilon}}{\partial v} f_{\varepsilon}+2 r_{\varepsilon} f_{\varepsilon},
$$

and as $\varepsilon \rightarrow 0$, we have

$$
\begin{aligned}
& g_{\varepsilon} \rightarrow \operatorname{sgn}(v) f=|f| \quad \text { in } L_{\mathrm{loc}}^{1}\left((0, \infty) \times \mathbb{R}^{n+1}\right), \\
& f_{\varepsilon}^{2} \rightarrow f^{2} \quad \text { in } L_{\mathrm{loc}}^{1}\left((0, \infty) \times \mathbb{R}^{n+1}\right), \\
& g_{\varepsilon}(t=0) \rightarrow\left|\chi\left(v, u^{0}(x)\right)\right| \quad \text { in } L^{1}\left(\mathbb{R}^{n+1}\right), \\
& f_{\varepsilon}^{2}(t=0) \rightarrow\left|\chi\left(v, u^{0}(x)\right)\right|^{2}=\left|\chi\left(v, u^{0}(x)\right)\right| \quad \text { in } L^{1}\left(\mathbb{R}^{n+1}\right)
\end{aligned}
$$

thanks to the continuity assumption at $t=0$. And for any nonnegative test function $\psi=\psi(x, v) \in \mathcal{D}\left(\mathbb{R}^{n+1}\right)$, for any $T>0$, we have 


$$
\begin{aligned}
\int_{\mathbb{R}^{n+1}}[ & \left.f_{\varepsilon}^{2}(T, x, v)-g_{\varepsilon}(T, x, v)\right] \psi(x, v) \mathrm{d} x \mathrm{~d} v \\
= & \int_{\mathbb{R}^{n+1}}\left[f_{\varepsilon}^{2}(t=0, x, v)-g_{\varepsilon}(t=0, x, v)\right] \psi(x, v) \mathrm{d} x \mathrm{~d} v \\
& +\int_{0}^{T} \int_{\mathbb{R}^{n+1}}\left[f_{\varepsilon}^{2}(t, x, v)-g_{\varepsilon}(t, x, v)\right] a(x, v) \cdot \nabla_{x, v} \psi(x, v) \mathrm{d} x \mathrm{~d} v \mathrm{~d} t \\
& +\int_{0}^{T} \int_{\mathbb{R}^{n+1}} m_{\varepsilon}(t, x, v)\left[\operatorname{sgn}_{\varepsilon}^{\prime}(v)-2 \partial_{v} f_{\varepsilon}\right] \psi(x, v) \mathrm{d} x \mathrm{~d} v \mathrm{~d} t \\
& +\int_{0}^{T} \int_{\mathbb{R}^{n+1}} m_{\varepsilon}(t, x, v)\left[\operatorname{sgn}_{\varepsilon}(v)-2 f_{\varepsilon}\right] \partial_{v} \psi(x, v) \mathrm{d} x \mathrm{~d} v \mathrm{~d} t \\
& +\int_{0}^{T} \int_{\mathbb{R}^{n+1}} r_{\varepsilon}(t, x, v)\left[2 f_{\varepsilon}-\operatorname{sgn}_{\varepsilon}(v)\right] \psi(x, v) \mathrm{d} x \mathrm{~d} v \mathrm{~d} t \\
& -\int_{0}^{T} \int_{\mathbb{R}^{n+1}} \operatorname{sgn}_{\varepsilon}^{\prime}(v) a_{n+1}(x, v) f_{\varepsilon}(t, x, v) \psi(x, v) \mathrm{d} x \mathrm{~d} v \mathrm{~d} t .
\end{aligned}
$$

Recall that

$$
\partial_{v} f_{\varepsilon}=(\delta(v)-v) * \varphi_{\varepsilon}(t, x, v)
$$

and that $\operatorname{sgn}_{\varepsilon}^{\prime}(v)=2 \delta(v) * \varphi_{\varepsilon}(t, x, v)$; hence

$$
\operatorname{sgn}_{\varepsilon}^{\prime}(v)-2 \partial_{v} f_{\varepsilon}=2 v * \varphi_{\varepsilon}(t, x, v) .
$$

Choose $\psi(x, v)=\theta\left(\frac{x}{R}\right) \phi_{R}(v)$, with $\theta \in \mathcal{D}\left(\mathbb{R}^{n}\right), \phi_{R} \in \mathcal{D}(\mathbb{R}), \phi_{R}(v)=1$ if $|v| \leqslant R, \phi_{R}(v)=0$ if $|v| \geqslant R+1, \theta(x)=1$ when $|x| \leqslant 1$, and $0 \leqslant\left|\phi_{R}^{\prime}\right| \leqslant 2(R>0$ arbitrary). Then for all $\varepsilon>0$, we have

$$
\begin{aligned}
\left|\int_{0} \int_{\mathbb{R}^{n+1}} m_{\varepsilon}(t, x, v)\left[\operatorname{sgn}_{\varepsilon}(v)-2 f_{\varepsilon}\right] \partial_{v} \psi(x, v) \mathrm{d} x \mathrm{~d} v \mathrm{~d} t\right| & \leqslant 2 \int_{0}^{T} \int_{\mathbb{R}^{n}} \int_{R \leqslant|v| \leqslant R+1} m_{\varepsilon}(t, x, v) \mathrm{d} x \mathrm{~d} v \mathrm{~d} t \\
& \leqslant 2 \int_{R \leqslant|v| \leqslant R+1} \sup \left(\mu_{T+\varepsilon}(v-\varepsilon), \mu_{T+\varepsilon}(v+\varepsilon)\right) \mathrm{d} v \\
& \leqslant 2\left(\mu_{T+1}(R-1)+\mu_{T+1}(-R+1)\right)
\end{aligned}
$$

for $R$ large enough, for $0<\varepsilon<1$.

We pass to the limit as $\varepsilon \rightarrow 0$ : for almost every $T>0$, we have

$$
\begin{aligned}
& \int_{\mathbb{R}^{n+1}}\left[f(T, x, v)^{2}-|f(T, x, v)|\right] \theta\left(\frac{x}{R}\right) \phi_{R}(v) \mathrm{d} x \mathrm{~d} v \\
& \geqslant \int_{0} \int_{\mathbb{R}^{n+1}}\left[f^{2}(t, x, v)-|f(t, x, v)|\right] a(x, v) \cdot \nabla_{x, v}\left(\theta\left(\frac{x}{R}\right) \phi_{R}(v)\right) \mathrm{d} x \mathrm{~d} v \mathrm{~d} t \\
& \quad+2 \limsup _{\varepsilon \rightarrow 0} \int_{0}^{T} \int_{\mathbb{R}^{n+1}} m_{\varepsilon}(t, x, v) v * \varphi_{\varepsilon}(t, x, v) \theta\left(\frac{x}{R}\right) \phi_{R}(v) \mathrm{d} x \mathrm{~d} v \mathrm{~d} t-2\left(\mu_{T+1}(R-1)+\mu_{T+1}(-R+1)\right) .
\end{aligned}
$$


We now pass to the limit as $R \rightarrow \infty$ using assumption (56):

$$
\begin{aligned}
0 & \geqslant \int_{\mathbb{R}^{n+1}}\left[f(T, x, v)^{2}-|f(T, x, v)|\right] \mathrm{d} x \mathrm{~d} v \\
& \geqslant \limsup _{R \rightarrow \infty} \limsup _{\varepsilon \rightarrow 0} \int_{0}^{T} \int_{\mathbb{R}^{n+1}} m_{\varepsilon}(t, x, v) 2 v * \varphi_{\varepsilon}(t, x, v) \theta\left(\frac{x}{R}\right) \phi_{R}(v) \mathrm{d} x \mathrm{~d} v \mathrm{~d} v \geqslant 0 .
\end{aligned}
$$

Consequently, $f(T, x, v)^{2}=|f(T, x, v)|$ a.e. and $f$ only takes the values $0, \pm 1$. Combining this property with (54) leads us to $f(t, x, v)=\chi(v, u(t, x))$ for some $u \in L_{\text {loc }}^{\infty}\left((0,+\infty), L^{1}\left(\mathbb{R}^{n}\right)\right)$.

Moreover,

$$
\limsup _{R \rightarrow \infty} \limsup _{\varepsilon \rightarrow 0} \int_{0}^{T} \int_{\mathbb{R}^{n+1}} m_{\varepsilon}(t, x, v) v * \varphi_{\varepsilon}(t, x, v) \theta\left(\frac{x}{R}\right) \phi_{R}(v) \mathrm{d} x \mathrm{~d} v \mathrm{~d} v=0
$$

and since $f=\chi(v, u(t, x)), v(t, x, v)=\delta(v-u(t, x))$, which proves the last assertion of Proposition 19.

The first part of Theorem 17 is thus proved. We are now ready to show the $L^{1}$ contraction principle for generalized kinetic solutions. Take two generalized solutions $f_{1}, f_{2}$, continuous at time $t=0$ and satisfying (56). Setting $f_{\varepsilon, i}=$ $f_{i} * \varphi_{\varepsilon}$, we have

$$
\partial_{t} f_{\varepsilon, i}+\operatorname{div}_{x, v}\left(a f_{\varepsilon, i}\right)=\frac{\partial m_{\varepsilon, i}}{\partial v}+r_{\varepsilon i}, \quad i=1,2,
$$

and $f_{i}(t=0)=\chi\left(v, u_{i}^{0}\right)$. It is easily checked that

$$
\partial_{t}\left(f_{\varepsilon, 1}-f_{\varepsilon, 2}\right)^{2}+\operatorname{div}_{x, v}\left(a\left(f_{\varepsilon, 1}-f_{\varepsilon, 2}\right)^{2}\right)=2 \frac{\partial\left(m_{\varepsilon, 1}-m_{\varepsilon, 2}\right)}{\partial v}\left(f_{\varepsilon, 1}-f_{\varepsilon, 2}\right)+2\left(r_{\varepsilon 1}-r_{\varepsilon 2}\right)\left(f_{\varepsilon, 1}-f_{\varepsilon, 2}\right) .
$$

Take a test function $\psi_{R}(x, v):=\theta\left(\frac{x}{R}\right) \phi_{R}(v)$, with $R>0$ and with $\theta, \phi_{R}$ satisfying the same hypotheses as in the proof of Proposition 19. Multiplying (60) by $\psi_{R}(x, v)$ and integrating on $(0, T) \times \mathbb{R}^{n+1}(T>0$ arbitrary) yields

$$
\begin{aligned}
& \int_{\mathbb{R}^{n+1}}\left(f_{\varepsilon, 1}-f_{\varepsilon, 2}\right)^{2}(t=T, x, v) \psi_{R}(x, v) \mathrm{d} x \mathrm{~d} v \\
& =\int_{\mathbb{R}^{n+1}}\left(f_{\varepsilon, 1}-f_{\varepsilon, 2}\right)^{2}(t=0, x, v) \psi_{R}(x, v) \mathrm{d} x \mathrm{~d} v \\
& \quad+\int_{0}^{T} \int_{\mathbb{R}^{n+1}}\left(f_{\varepsilon, 1}-f_{\varepsilon, 2}\right)^{2} a(x, v) \cdot \nabla_{x, v} \psi_{R}(x, v) \mathrm{d} x \mathrm{~d} v \mathrm{~d} t \\
& \quad-2 \int_{0 \mathbb{R}^{n+1}}^{T}\left(m_{\varepsilon, 1}-m_{\varepsilon, 2}\right)\left(f_{\varepsilon, 1}-f_{\varepsilon, 2}\right) \theta\left(\frac{x}{R}\right) \partial_{v} \phi_{R}(v) \mathrm{d} x \mathrm{~d} v \mathrm{~d} t \\
& \quad-2 \int_{0 \mathbb{R}^{n+1}}^{T}\left(m_{\varepsilon, 1}-m_{\varepsilon, 2}\right)\left(\partial_{v} f_{\varepsilon, 1}-\partial_{v} f_{\varepsilon, 2}\right) \psi_{R}(x, v) \mathrm{d} x \mathrm{~d} v \mathrm{~d} t \\
& \quad+2 \int_{0}^{T} \int_{\mathbb{R}^{n+1}}\left(r_{\varepsilon 1}-r_{\varepsilon 2}\right)\left(f_{\varepsilon, 1}-f_{\varepsilon, 2}\right) \psi_{R}(x, v) \mathrm{d} x \mathrm{~d} v \mathrm{~d} t .
\end{aligned}
$$

As in the proof of Proposition 19, first we let $\varepsilon$ go to 0 , and then $R \rightarrow \infty$. Then: 
- (61) goes to

$$
\int_{\mathbb{R}^{n+1}}\left|f_{1}-f_{2}\right|^{2}(T, x, v) \mathrm{d} x \mathrm{~d} v=\int_{\mathbb{R}^{n+1}}\left|f_{1}-f_{2}\right|(T, x, v) \mathrm{d} x \mathrm{~d} v
$$

for almost every $T>0$ since $f_{i}=\chi\left(v, u_{i}\right)$, and (62) goes to

$$
\int_{\mathbb{R}^{n+1}}\left|f_{1}-f_{2}\right|^{2}(t=0, x, v) \mathrm{d} x \mathrm{~d} v=\int_{\mathbb{R}^{n+1}}\left|f_{1}-f_{2}\right|(t=0, x, v) \mathrm{d} x \mathrm{~d} v
$$

thanks to the continuity assumption at time $t=0$.

- (63) goes to 0 according to assumptions (3), (56).

- (64) is bounded uniformly in $\varepsilon$ by

$$
2\left[\sup \left(\mu_{T+1}^{1}(R-1), \mu_{T+1}^{1}(-R+1)\right)+\sup \left(\mu_{T+1}^{2}(R-1), \mu_{T+1}^{2}(-R+1)\right)\right] \underset{R \rightarrow \infty}{\longrightarrow} 0
$$

where $\mu_{T}^{i}$ is the function that occurs in the bound (52) for $m_{i}$.

- (66) goes to 0 as $\varepsilon \rightarrow 0$ for each $R>0$ according to Lemma 18 .

- In (65), we have

$$
\begin{aligned}
\partial_{v} f_{\varepsilon, 1}-\partial_{v} f_{\varepsilon, 2} & =\left[\delta(v) * \varphi_{\varepsilon}-\delta\left(v-u_{1}(t, x)\right) * \varphi_{\varepsilon}\right]-\left[\delta(v) * \varphi_{\varepsilon}-\delta\left(v-u_{2}(t, x)\right) * \varphi_{\varepsilon}\right] \\
& =\delta\left(v-u_{2}(t, x)\right) * \varphi_{\varepsilon}-\delta\left(v-u_{1}(t, x)\right) * \varphi_{\varepsilon} .
\end{aligned}
$$

Hence (65) is equal to

$$
\begin{aligned}
& 2 \int_{0}^{T} \int_{\mathbb{R}^{n+1}}\left(m_{\varepsilon, 1}-m_{\varepsilon, 2}\right)\left(\delta\left(v-u_{1}\right) * \varphi_{\varepsilon}-\delta\left(v-u_{2}\right) * \varphi_{\varepsilon}\right) \psi_{R}(x, v) \mathrm{d} x \mathrm{~d} v \mathrm{~d} t \\
& \quad \leqslant 2 \int_{0}^{T} \int_{\mathbb{R}^{n+1}}\left(m_{\varepsilon, 1} \delta\left(v-u_{1}\right) * \varphi_{\varepsilon}+m_{\varepsilon, 2} \delta\left(v-u_{2}\right) * \varphi_{\varepsilon}\right) \psi_{R}(x, v) \mathrm{d} x \mathrm{~d} v \mathrm{~d} t
\end{aligned}
$$

and the right-hand side of the above inequality goes to 0 as $\varepsilon \rightarrow 0$ for all $R>0$ according to Proposition 19 .

Consequently, passing to the limit in (61)-(66) yields

$$
\int_{\mathbb{R}^{n+1}}\left|f_{1}-f_{2}\right|(T, x, v) \mathrm{d} x \mathrm{~d} v \leqslant \int_{\mathbb{R}^{n+1}}\left|f_{1}-f_{2}\right|(t=0, x, v) \mathrm{d} x \mathrm{~d} v
$$

for almost every $T>0$, which is the desired inequality.

\section{References}

[1] C.M. Dafermos, Hyperbolic Conservation Laws in Continuum Physics, Grundlehren Math. Wiss., vol. 325, Springer-Verlag, Berlin, 1999.

[2] R. DiPerna, Measure-valued solutions to conservation laws, Arch. Rational Mech. Anal. 88 (1985) 223-270.

[3] S.N. Kruzkhov, Generalized solutions of the Cauchy problem in the large for nonlinear equations of first order, Soviet Math. Dokl. 10 (1969).

[4] S.N. Kruzkhov, First order quasilinear equations in several independent variables, Math. USSR-Sb. 10 (1970) $217-243$.

[5] P.-L. Lions, B. Perthame, E. Tadmor, Formulation cinétique des lois de conservation scalaires multidimensionnelles, C. R. Acad. Sci. Paris, Sér. I 312 (1991) 97-102.

[6] P.-L. Lions, B. Perthame, E. Tadmor, A kinetic formulation of multidimensional conservation laws and related equations, J. Amer. Math. Soc. 7 (1994) 169-191.

[7] B. Perthame, Kinetic Formulation of Conservation Laws, Oxford Lecture Series in Math. Appl., vol. 21, Oxford University Press, New York, 2002.

[8] B. Perthame, Uniqueness and error estimates in first order quasilinear conservation laws via the kinetic entropy defect measure, J. Math. Pures Appl. 77 (1998) 1055-1064. 
[9] B. Perthame, E. Tadmor, A kinetic equation with kinetic entropy functions for scalar conservation laws, Comm. Math. Phys. 136 (1991) 501-517.

[10] D. Serre, Systèmes de lois de conservation I et II, Diderot Editeur, Arts et Sciences, 1996.

[11] A. Szepessy, An existence result for scalar conservation laws using measure valued solutions, Comm. Partial Differential Equations 14 (1989) 1329-1349. 